\title{
Improved atomization, collision and sub-grid scale momentum coupling models for transient vaporizing engine sprays
}

\author{
Federico Perini ${ }^{\mathrm{a}, *}$, Rolf D. Reitz ${ }^{\mathrm{a}}$ \\ ${ }^{a}$ University of Wisconsin-Madison, 1500 Engineering Drive, Madison, WI 53706
}

\begin{abstract}
A computationally efficient spray model is presented for the simulation of transient vaporizing engine sprays. It is applied to simulate high-pressure fuel injections in a constant volume chamber and in mixture preparation experiments in a light-duty internal combustion engine. The model is based on the LagrangianParticle/Eulerian-Fluid approach, and an improved blob injection model is used that removes numerical dependency on the injected number of computational parcels. Atomization is modeled with the hybrid Kelvin-Helmholtz/RayleighTaylor scheme, in combination with a drop drag model that includes Mach number and Knudsen number effects. A computationally efficient drop collision scheme is presented, tailored for large numbers of parcels, using a deterministic collision impact definition and kd-tree data search structure to perform radius-of-influence based, grid-independent collision probability estimations. A near-nozzle sub-grid scale flow-field representation is introduced to reduce numerical grid dependency, which uses a turbulent transient gas-jet model with a Stokes-Strouhal analogy assumption. An implicit coupling method was developed for the Arbitrary Lagrangian-Eulerian (ALE) turbulent flow solver. A multi-objective genetic algorithm was used to study the interactions of the various model constants, and to provide an optimal calibration. The optimal set showed similar values of the primary breakup constants as values used in the lit-
\end{abstract}

\footnotetext{
${ }^{*}$ Corresponding author. Phone: +1-608-515-0186

Email address: perini@wisc.edu (Federico Perini)
}

Preprint submitted to Elsevier

September 19, 2015 
erature. However, different values were seen for the gas-jet model constants for accurate simulations of the initial spray transient. The results show that there is a direct correlation between the predicted initial liquid-phase transient and the global gas-phase jet penetration. Model validation was also performed in engine simulations with the same set of constants. The model captured mixture preparation well in all cases, proving its suitability for simulations of transient spray injection in engines.

Keywords: spray, transient, atomization, turbulent gas-jet, collision, Stokes, RANS, Spray A

\section{Introduction}

The controllability of low-temperature combustion strategies in advanced internal combustion engines relies on local mixture preparation, which is typically achieved by optimized fuel injection (Musculus et al. (2013)), which produces

5 reactivity gradients that sustain a robust ignition event, surrounded by a lean and low-temperature mixture (Reitz and Duraisamy (2015)). The fuel spray characteristics affect the air-fuel mixture formation by means of interacting processes:

- atomization, which affects momentum transfer to the gas phase through a specific spray cone angle and drop size distribution;

- turbulent air entrainment, produced by the injected fuel's momentum exchange and by the local flow field;

- vaporization, which converts the liquid phase into a gaseous mixture.

Recent high resolution experiments and high-performance computational modeling studies highlight the transient nature of these mechanisms, and how the interactions among them affect spray combustion Gorokhovski and Herrmann (2008a). Aggarwal and Sirignano (1985) used a Lagrangian-particle/EulerianFluid (LDEF) approach to simulate unsteady flame propagation from a liquid spray jet. The effects of partially- and fully- resolved turbulence calculations 
on turbulent spray simulations have been studied by Bellan (2000); Miller and Bellan (2000); Okongo and Bellan (2000, 2004); Leboissetier et al. (2005). Pera et al. (2006) studied spray combustion using large eddy simulations (LES), highlighting how sub-grid scale (sgs, i.e., unresolved) mixture fraction distributions affect the accuracy of highly resolved simulations. Tsang et al. (2014) compared LES simulations of turbulent spray jets in combination with a Lagrangian spray particle model, and observed that sub-grid scale layer mixing significantly determines the overall jet structure. Sankaran and Menon (2002) have shown via LES that when the fuel spray is injected in a swirling environment, droplet dispersion is dramatically increased by stretching of the large-scale vortex structure.

30 A number of studies have also used direct numerical simulation (DNS) to study the local transient structure of turbulent reacting and non-reacting spray jets, and the formation of alternate premixed and diffusion flame combustion regions (Domingo et al. (2005); Reveillon et al. (1998); Reveillon and Vervisch (2005); Luo et al. (2011)).

35 Pickett et al. (2011) have extensively measured spray injection and mixture formation at high pressures in a constant volume vessel using schlieren and Rayleigh scattering imaging, providing experimental spray data (e.g., the Engine Combustion Network 'Spray A' case), and showing how a zero-dimensional spray model can capture mixture formation correctly if its geometrical structure is properly initialized. Sahoo et al. (2011, 2012, 2013); Miles et al. (2013); Perini et al. (2013) have presented mixture preparation imaging using planar laser-induced fluorescence (PLIF) of full and pulsed injections in a light-duty optical engine, showing significant differences in mixture formation for transient injections.

45 In order to capture fuel spray dynamics in internal combustion engine simulations, the Lagrangian-Particle/Eulerian-Fluid (LDEF, Dukowicz (1980)) approach is commonly adopted because of the many scales separating the internal injector and near-nozzle flows that affect the liquid phase development, and the consequent gas-phase turbulent flame in the combustion chamber. Because of 50 the lack of resolution in the description of the liquid spray core, phenomeno- 
logical atomization models have been developed (e.g., Reitz and Bracco (1982); Reitz (1987); Reitz and Diwakar (1987); Tanner (1997); Habchi et al. (1997); Huh et al. (1998); Bianchi and Pelloni (1999); Beale and Reitz (1999); Hiroyasu (2000); Gorokhovski and Herrmann (2008b)), within the LDEF framework.

55 This approach, although successful in a wide variety of simulations, suffers from significant time-step and grid-resolution dependency, which is especially true for Reynolds-Averaged Navier-Stokes (RANS) approaches where all turbulence scales of the flow are modeled, and the grid resolution is more than one order of magnitude coarser than the characteristic injector diameter. Thus, some recent approaches have attempted to reduce the dependency of spray simulations on grid resolution and model constants by applying subcycling schemes to the Lagrangian particle step (Wang et al. (2010)), using region-of-interest instead than Computational Fluid Dynamics (CFD) grid-based collision calculations (Schmidt and Rutland (2004); Munnannur and Reitz (2009)), and using model-computed instantaneous field velocities in the near-nozzle region instead of the under-resolved CFD flow fields (Ra et al. (2005); Abani et al. (2008a,b)). The last approach makes use of predictions from the theory of turbulent round jets (see, for example, Islam and Tucker (1980); Bremhorst and Hollis (1990); Abraham (1996); Iyer and Abraham (1997); Song and Abraham (2003); Singh 70 and Musculus (2010); Musculus (2009); Liepmann and Gharib (1992)). These models can predict a turbulent jet's penetration, velocity profiles, and gas concentrations within the jet, based on parameters such as an effective diameter, the densities of the gaseous environment, and the time-varying injection velocity. The models provide relevant flow properties that would require much higher resolution in engineering combustor simulations, similar to what is commonly done to model near-wall viscous boundary layers (e.g., the logarithmic law-of-the-wall).

In this work, a new spray model for Lagrangian-Particle/Eulerian-Fluid solvers is presented and applied to simulate high-pressure transient fuel sprays.

so The model features an improved blob injection model with the hybrid KelvinHelmholtz/Rayleigh-Taylor atomization scheme (Beale and Reitz (1999)), where 
the assumption of a computational parcel containing multiple blobs is replaced by a fully-resolved, one-to-one blob to parcel representation. A drop collision scheme, tailored for large numbers of parcels, is used which exploits a new deterministic collision impact estimation obtained from a kd-tree representation of the spray jet, and the extended collision outcomes of Munnannur and Reitz (2007). The spray model dynamics are computed using the sub-grid scale gas-jet model of Abani and Reitz (2007), under a Stokes-Strouhal analogy assumption for the liquid phase, which is coupled with an Arbitrary Lagrangian-Eulerian 90 (ALE) turbulent flow solver (Torres and Trujillo (2006)) in an implicit fashion. A study of the effects of the resulting model constants was carried out by means of a multi-objective genetic algorithm, and an optimal calibration for accurate transient liquid and vapor-phase properties in constant-volume sprays was found. The optimal set of constants was then applied to mixture preparation simulations in a light-duty combustion engine operating a low-load, slightly boosted operating condition, representing a partially-premixed combustion (PPC). The validation verifies the accuracy of the model and its suitability for simulations of transient spray injections in engines and other practical combustion systems. Guidance is also given for calibration of the model constants outside of the current ranges.

The paper is structured as follows. The first part describes the model equations and the improvements introduced over existing models. The second part deals with the sub-grid scale unsteady gas-jet superposition model. The third part summarizes the setup and the results of the Genetic Algorithm (GA)-based optimization of the model constants. The last section provides the model validation with respect to constant-volume and engine injection experiments.

\section{Model description}

\subsection{Blob injection model}

An improved blob injection model was used, in combination with the hybrid Kelvin-Helmholtz/Rayleigh-Taylor breakup model, as reported in Figure 
1. In the original blob injection model by Reitz and Diwakar (1987), a set of computational parcels, representing 'blobs', or portions of the injected liquid column, is injected into the multidimensional domain. Each blob is represented as a sphere and characterized by an initial diameter equal to an effective nozzle diameter $d_{n o z}$, greater than or equal to the diameter $d_{i n j}$ of the vena contracta within the nozzle:

$$
d_{i n j}=\sqrt{c_{D}} d_{n o z},
$$

where $c_{D}$ is a nozzle discharge coefficient. In a typical spray simulation, a fixed number of parcels of identical drops is injected, each parcel carrying a blob number density representing the average behavior of a number of blobs. This approach was useful to prevent an excessive number of computational parcels from being generated during breakup, however, it introduces model dependency on calibration parameters. Hence, in the present work, a dynamically allocated spray injection approach was developed, with only one blob per computational parcel. At the injection, the requested number of blobs $n_{p, i n j}$ per injection event is computed as:

$$
n_{p, i n j}=\frac{m_{i n j}}{\frac{\pi}{6} \rho_{p} d_{i n j}^{3}},
$$

where $m_{i n j}$ is the injected fuel mass and $\rho_{p}$ the fuel density at injection. An initial parcel space is allocated and dynamically adjusted during the simulation based on changes in the total parcel number due to breakup and evaporation events, thus keeping memory allocation efficient.

In the blob injection model the user must also specify an initial spray cone angle, $\theta_{i n j}$. While this assumption is useful when internal injector characteristics are not known, Pickett et al. (2011) highlighted how the spray cone angle is time-varying, and is the parameter that mostly affects mixture distribution properties in high-pressure environment sprays. Hence, the relationship by Reitz 
and Bracco (1979), for sharp-edge nozzles, was introduced:

$$
\tan \left(\frac{\theta_{i n j}}{2}\right)=\frac{2 \sqrt{3} \pi}{3\left(3+0.28 l_{n o z} / d_{n o z}\right)} \sqrt{\frac{\rho_{g}}{\rho_{l}}},
$$

where $l_{n o z} / d_{n o z}$ is the internal nozzle length/diameter ratio, $\rho_{l}$ is the liquid fuel density and $\rho_{g}$ is the (time-varying) gas density.

\subsection{Kelvin-Helmholtz/Rayleigh-Taylor hybrid breakup model}

The hybrid Kelvin-Helmholtz/Rayleigh-Taylor (KH-RT) model by Reitz (1987) was adopted. Primary breakup follows the KH mode, representing shedding of drops from the blob bulk by means of shear stresses. New, child droplets are progressively stripped from the blob with sizes equal to a stable breakup radius $r_{K H}$,

$$
r_{K H}=C_{\Lambda K H} \Lambda_{K H}
$$

and the parent blob is shrunk according to an exponential decay rate if the stable breakup radius is smaller than the blob size,

$$
\frac{d r_{p}}{d t}=-\frac{r_{p}-r_{K H}}{\tau_{K H}}, \text { where } \tau_{K H}=\frac{3.788 B_{1} r_{p}}{\Omega_{K H} \Lambda_{K H}},
$$

and $C_{\Lambda K H}=0.61$ and $B_{1}$ is a model constant (to be described below), and

$$
\begin{aligned}
& \Omega_{K H}=\frac{0.34+0.385 W e_{g}^{1.5}}{\left(1+O h_{l}\right)\left(1+1.4 T^{0.6}\right)} \sqrt{\frac{\sigma}{\rho_{l} r_{p}}}, \\
& \Lambda_{K H}=9.02 r_{p} \frac{\left(1+0.45 \sqrt{O h_{l}}\right)\left(1+0.4 T^{0.7}\right)}{\left(1+0.865 W e_{g}^{1.67}\right)^{0.6}}
\end{aligned}
$$

are the frequency and wavelength of the fastest $\mathrm{KH}$ wave mode growing on the blob's surface. $O h_{l}=\sqrt{W e_{l}} / R e_{l}$ is the Ohnesorge, $T=O h_{l} \sqrt{W e_{g}}$ the Taylor number, and $W e_{g}=\rho_{g}\|\boldsymbol{\theta}-\mathbf{u}\|^{2} r / \sigma$ is the Weber number for the liquid $(l)$ and gas phase $(g)$, respectively; $\boldsymbol{\theta}$ and $\mathbf{u}$ are the drop and the gas velocities, respectively; $\sigma$ is the drop surface tension. If the stable breakup radius is larger than the blob radius $\left(r_{K H}>r_{p}\right)$, instead, the blob size is rearranged based 
on the effect of either jet disturbance frequency or the volume contained in a surface wave:

$$
r_{p}=\sqrt[3]{\min \left\{\frac{3 \pi}{2 \Lambda_{K H}} r_{p}^{2}\|\boldsymbol{\theta}-\mathbf{u}\|, \frac{3}{4} r_{p}^{2} \Lambda_{K H}\right\}} .
$$

The mass shed from the parent blob is accumulated over time, and a new child parcel is only generated when a user specified amount of mass is accumulated:

$$
m_{\text {child }} \geq \frac{4}{3} \pi f_{K H \text { brth }} \rho_{p} N_{p} r_{p}^{3},
$$

$f_{K H b r t h}$ being another model constant usually set at $3 \%$.

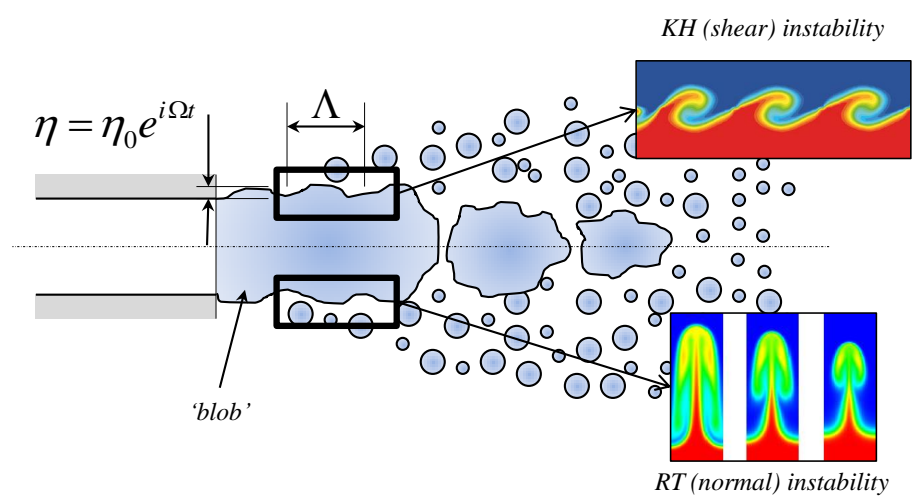

Figure 1: Atomization mechanism via the hybrid Kelvin-Helmholtz/Rayleigh-Taylor (KH-RT) instability model

Breakup from Rayleigh-Taylor (RT) instability represents a catastrophic event that converts the blob into a large number of tiny drops. According to this mode, the fastest growing wave $\Omega_{R T}$ due to normal acceleration at the drop-gas interface is

$$
\begin{aligned}
\Lambda_{R T} & =C_{\Lambda R T} \frac{\pi}{K_{R T}}, \quad K_{R T}=\sqrt{\frac{\left|g_{t}\left(\rho_{l}-\rho_{g}\right)\right|}{3 \sigma}}, \\
\Omega_{R T} & =\sqrt{\frac{2}{\sqrt{27 \sigma}} \frac{\left|g_{t}\left(\rho_{l}-\rho_{g}\right)\right|^{3 / 2}}{\rho_{l}+\rho_{g}}}
\end{aligned}
$$


where $g_{t}=\left\langle\mathbf{g}+\dot{\boldsymbol{\theta}}, \frac{\boldsymbol{\theta}}{\|\boldsymbol{\theta}\|}\right\rangle$ is the acceleration in the direction of travel, including gravity (g) effects, and $C_{\Lambda R T}$ is a model constant. Catastrophic breakup occurs after the lifetime of the growing RT wave exceeds the characteristic timescale

$$
\tau_{R T}=\frac{C_{\tau R T}}{\Omega_{R T}},
$$

where $C_{\tau R T}$ is a model constant. In order to prevent RT breakup from occurring too early during the injection, the breakup length criterion introduced by Beale and Reitz (1999) such that no RT breakup takes place earlier than reaching Levich's breakup length $L_{b}$ downstream of the nozzle:

$$
L_{b}=C_{b} \sqrt{\pi \frac{d_{i n j}^{2}}{4} \frac{\rho_{l}}{\rho_{g}}},
$$

170 an aerodynamic drag force, which defines the amount of momentum transferred from the liquid to the gas-phase when a relative velocity exists. Drag is modeled using the formulation of Amsden et al. (1989):

$$
\mathbf{F}_{D}=\frac{3}{8} \frac{\rho_{g}}{\rho_{l}} C_{D} \frac{\left\|\mathbf{u}+\boldsymbol{\theta}^{t}-\boldsymbol{\theta}\right\|}{r_{p}}\left(\mathbf{u}+\boldsymbol{\theta}^{t}-\boldsymbol{\theta}\right)=d_{p}\left(\mathbf{u}+\boldsymbol{\theta}^{t}-\boldsymbol{\theta}\right),
$$

where $C_{D}$ is the sphere drag coefficient, and $\boldsymbol{\theta}^{t}$ is a turbulent parcel dispersion velocity randomly chosen from a Gaussian distribution $G\left(\boldsymbol{\theta}^{t}\right)$ with standard deviation $2 / 3 k_{t}$ computed using the model of Amsden et al. (1989):

$$
G\left(\boldsymbol{\theta}^{t}\right)=\sqrt{\frac{3}{4 \pi k_{t}}} \exp \left(-\frac{3}{4 k_{t}}\left\|\boldsymbol{\theta}^{t}\right\|^{2}\right)
$$


where $k_{t}$ represents turbulence kinetic energy. A Reynolds- and Mach- dependent implementation of the drag coefficient function was introduced, which makes use of a widely adopted correlation for Reynolds number dependency of the sphere drag coefficient $(f(R e)$, Morrison (2013)), corrected for Mach number dependency using data from Miller and Bailey (1979), interpolated using a two-dimensional Bezier function $(g(M a, R e)$, Collins (2014)):

$$
\begin{aligned}
C_{D}(R e, M a) & =f(R e) g(M a, R e), \\
f(R e) & =\frac{24}{R e}+\frac{2.6 a R e}{1+(a R e)^{1.52}}+\frac{0.411(b R e)^{-7.94}}{1+(b R e)^{-8}}+c\left(R e^{0.8}\right),
\end{aligned}
$$

where $a=0.2, b=263000, c=1 / 461000$. The adopted drag coefficient correlation is plotted in Figure 2, along with data points from a high-pressure spray injection simulation. Mach dependency appears to be negligible when the parcels are injected in no cross-flow. However, a significant number of cases in the range $R e \in\left[5 \cdot 10^{2}, 5 \cdot 10^{3}\right]$ is seen, where the formulation of Amsden et al. (1989) underestimates the drag coefficient up to a factor of $\approx 3$.

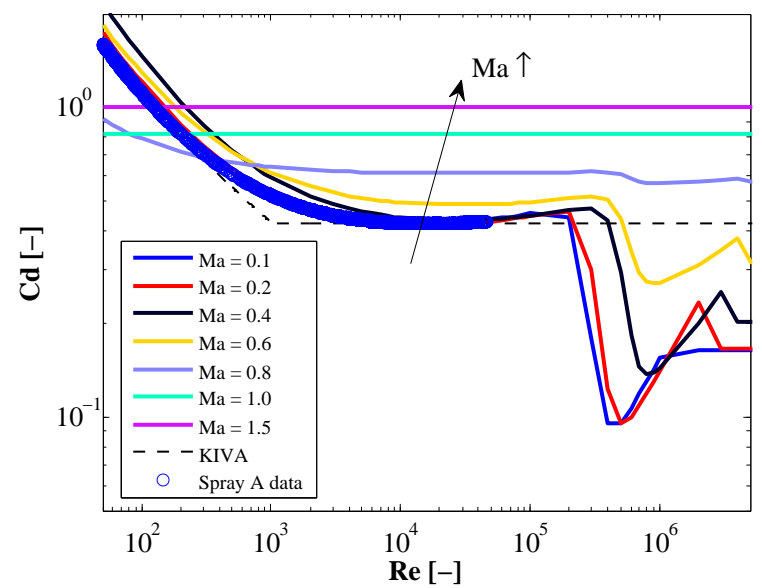

Figure 2: Comparison of adopted Mach-dependent sphere drag formulation versus KIVA (Amsden et al. (1989)). Circles represent parcel statuses during a high-pressure injection event of (Pickett et al. (2011)).

190 
Furthermore, the effects of drop size on drag coefficient were accounted for too using the correlation by Cunningham (1910), which models the deviation from Stokes law by means of a correction factor when a particle is moving in a fluid in a non-negligible Knudsen regime $(K n>\epsilon)$. The correction factor reduces the drag force by incorporating slip effects:

$$
C_{c}=1+\frac{\lambda}{r_{p}}\left(A_{1}+A_{2} \exp \left(-A_{3} r_{p} / \lambda\right)\right)
$$

where $\lambda$ is the gas molecular free path and $A_{1}=1.257, A_{2}=0.4, A_{3}=1.10$. A final multiplier to account for non-sphericity effects on large drops is applied, according to the distortion differential equation of Liu et al. (1993) which solves for the droplet distortion parameter $y$,

$$
\ddot{y}=\frac{2}{3} \frac{\rho_{g}}{\rho_{l}} \frac{\left\|\mathbf{u}+\boldsymbol{\theta}^{t}-\boldsymbol{\theta}\right\|^{2}}{r_{p}}-\frac{8 \sigma}{\rho_{l} r_{p}^{3}} y-\frac{5 \mu_{l}}{\rho_{l} r_{p}^{2}} \dot{y},
$$

In each 'computational parcel' only average properties of the contained ensemble of drops are stored (SMR, number of drops, density, etc.), but no spatial information on how the drops are displaced is given. Hence, to evaluate the collision probability between drops in two parcels, it is necessary to estimate 215

where $\mu_{l}$ is the internal drop viscosity, and represents a spring-mass system forced by the aerodynamic drag force, restored by surface tension and damped by the drop's liquid viscosity; the enhanced drag coefficient due to the drop's non sphericity $(y \neq 0)$ is given by $1+2.632 y$.

\subsection{Radius-of-Influence extended droplet Collision model}

Determination of potential collision partners can be the most computationally demanding task in spray modeling. Hence, a new droplet collision model was developed using the Radius-Of-Influence method of (Munnannur and Reitz (2009)). The outcome of a collision was modeled using the formulation by Munnannur and Reitz (2007, 2009). the volume of the cloud (volume-of-influence, VOI, or its radius-of-influence, 
ROI) that each parcel represents. According to Munnannur and Reitz (2009), a radius-of-influence is 'the radial distance around a parcel within which a potential collision partner is located'. In our approach, the radius-of-influence is the physical region occupied by a parcel's drop cloud. ROI was hence defined for each computational parcel $p$ as the spherical volume containing all $N_{p}$ droplets belonging to it. The liquid/gas volume fraction calculation in the region surrounding the parcel can be an expensive computational task, as it requires summation over all surrounding particles to evaluate the local average drop number density. Also, in a dense spray using the global liquid volume fraction as representative of each parcel is still a simplifying assumption; also still being grid-dependent. Hence, in this work, the volume-of-influence calculation was based on the hypothesis that all the droplets are equally far from each other within the parcel cloud, i.e., they are located at the vertices of regular tetrahedra, as reported in Figure 4 . The edges - or droplet center-to-center distances $-d_{c}$ are expressed as a multiple of the droplet radius: $d_{c}=k_{V} r_{p}$, where $k_{V}$ represents a parcel's volumetric expansion constant assumed to be equal to 10. This tetrahedralization approach is still based on the trade-off of calibrating for an appropriate $k_{V}$. However, it is extremely fast because of the purely analytical formulation; it also completely removes grid-dependency in the par235 cel's VOI calculation. $k_{V}$ is bound in $[2,+\infty)$ as $k_{V}=2$ means that all drops touch each other. Increasing the value of $k_{V}$ will spread the drops in a larger volume, increasing the number of potentially colliding parcels, but reducing the probability of collisions among drops of two colliding parcels (cf. Equation 26). Hence, the calibration for $k_{V}$ was determined as the minimum suitable value at the Pareto front that maximizes the number of collisions while minimizing each parcel's VOI. The Pareto front can be observed looking at predicted numbers of collisions for a non-breaking-up, non-vaporizing spray simulation with only the collision model active, as shown in Figure 3.

The VOI representation corresponds to a virtual tetrahedralization where all tetrahedra are regular, with edge length $d_{c}$ : the advantage of this assumption is that it is possible to compute the gas-liquid volume ratio with no need for 


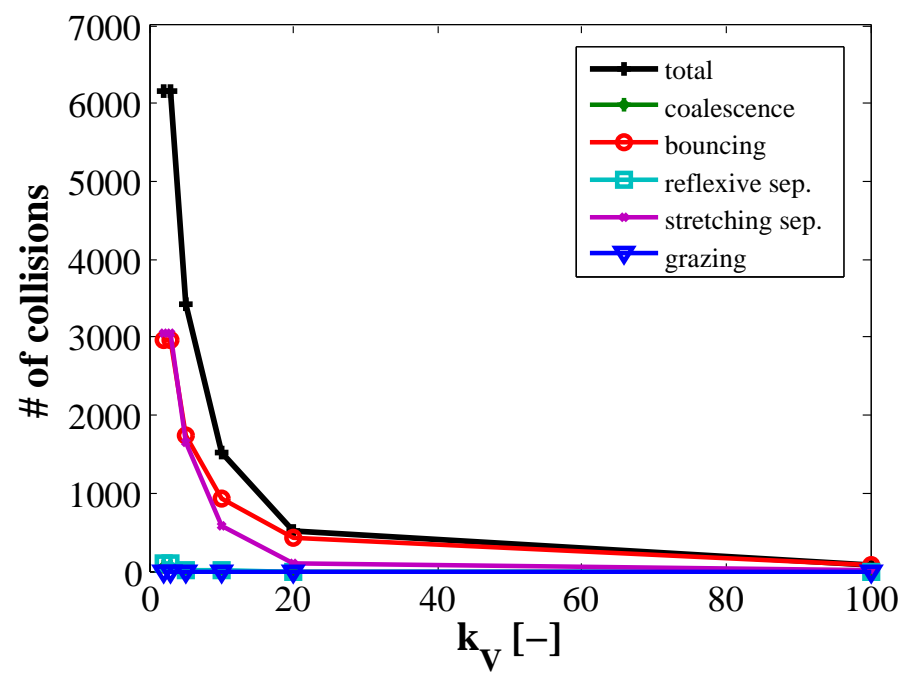

Figure 3: Dependency of predicted number of collisions versus volume-of-influence volumetric constant, $k_{V}$, for a non-breaking-up, non-vaporizing dense spray simulation.

actually building a tetrahedral grid. In a regular tetrahedron, all faces are equilateral triangles, and its volume can be expressed in terms of the edge length:

$$
V_{t e t}=\frac{d_{c}^{3}}{6 \sqrt{2}}
$$

Part of the tetrahedron volume is occupied by the liquid drops at each vertex. The regular tetrahedron's solid angle defines the number of virtual tetrahedra each drop shares its liquid volume with:

$$
n_{t e t}=\frac{4 \pi}{\cos ^{-1}(23 / 27)}
$$

Hence, the residual gaseous volume in each tetrahedron is

$$
V_{\text {gas }, \text { tet }}=\frac{\left(k_{V} r_{p}\right)^{3}}{6 \sqrt{2}}-\frac{4}{n_{\text {tet }}}\left(\frac{4 \pi}{3} r_{p}^{3}\right)
$$

It follows that the ratio of gas volume per liquid drop volume in each tetrahedron - and in the whole cloud - is:

$$
\frac{V_{\text {gas }}}{V_{d}}=\frac{n_{\text {tet }} V_{\text {gas }, \text { tet }}}{4 V_{d}}=\frac{1}{4 \cos ^{-1}(23 / 27)}\left[\frac{k_{V}^{3}}{2 \sqrt{2}}-\cos ^{-1}(23 / 27)\right] .
$$


Eventually, the parcel's volume of influence (VOI) for a tetrahedral cloud of $N_{p}$ drops is

$$
\begin{aligned}
V O I_{p} & =\frac{N_{p} r_{p}^{3}}{12 \cos ^{-1}(23 / 27)}\left[\frac{k_{V}^{3}}{2 \sqrt{2}}-\cos ^{-1}(23 / 27)\right], \\
\text { and } R O I_{p} & =\sqrt[3]{\frac{3}{4 \pi} V O I_{p}} .
\end{aligned}
$$

In order to establish the probability of collision among two parcels the collision frequency between the drops contained in two potentially colliding particles must be estimated (O'Rourke (1981)). Denoting the two parcels as $S$ (smaller drops) and $L$ (larger drops), the number of probable drop collisions in a parcelparcel collision event within a time-step is given by:

$$
n_{c o l}=\frac{\pi}{4} \frac{\left(R O I_{S}+R O I_{L}\right)^{2}}{V O I_{S}+V O I_{L}}\left|\boldsymbol{\theta}_{S}-\boldsymbol{\theta}_{L}\right|
$$
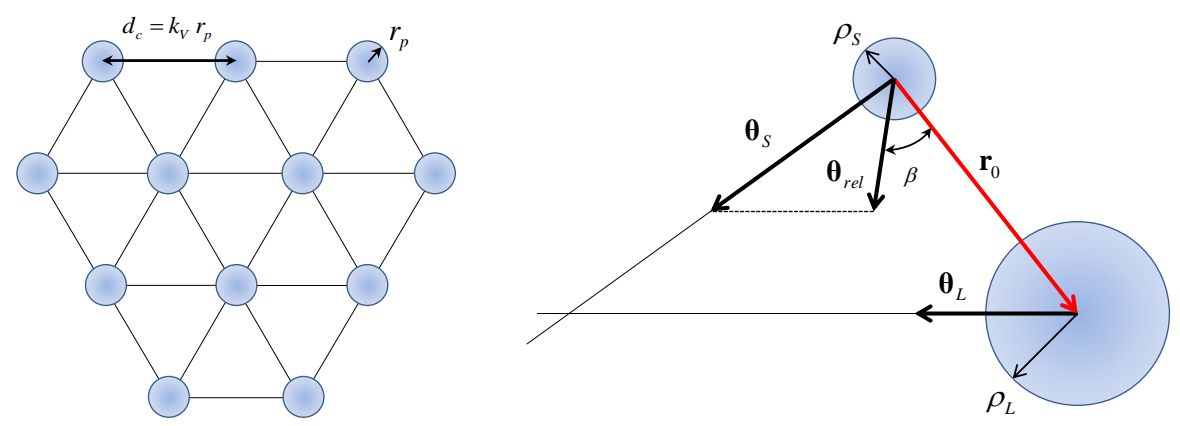

Figure 4: (left) regular tetrahedral grid displacement of drops in a computational parcel (2D example); (right) Schematic of binary collision impact parameter definition.

Simplifying assumptions such as requiring the parcels to lie within the same cell (such as in O'Rourke (1981)) are not viable for practical engineering applications on complex grids because of the extreme grid dependency. Thus, a new deterministic approach was developed, as reported in Figure 4. A parabolic law can be derived to describe the variation of distance $d$ between colliding drops within a time-step, having positions $\mathbf{x}_{S}$ and $\mathbf{x}_{L}$, under the assumption that parcel velocities $\boldsymbol{\theta}_{S}, \boldsymbol{\theta}_{L}$ are constant within the time-step: 


$$
\begin{aligned}
d^{2}\left(\mathbf{x}_{S}(t), \mathbf{x}_{L}(t)\right) & =\left\|\left(\mathbf{x}_{S 0}+t \boldsymbol{\theta}_{S}\right)-\left(\mathbf{x}_{L 0}+t \boldsymbol{\theta}_{L}\right)\right\|^{2} \\
& =p_{a} t^{2}+p_{b} t+p_{c},
\end{aligned}
$$

where

$$
\begin{aligned}
p_{a} & =\left\|\boldsymbol{\theta}_{S}-\boldsymbol{\theta}_{L}\right\|^{2}, \\
p_{b} & =2\left\langle\mathbf{x}_{S 0}-\mathbf{x}_{L 0}, \boldsymbol{\theta}_{S}-\boldsymbol{\theta}_{L}\right\rangle, \\
p_{c} & =\left\|\mathbf{x}_{S 0}-\mathbf{x}_{L 0}\right\|^{2} .
\end{aligned}
$$

Since $p_{a} \geq 0$, the parabolic cavity is upwards and the vertex coordinates provide the time and square distance value at which the two parcels are closest $t_{\min }=$ $-p_{b} /\left(2 p_{a}\right), d_{\min }^{2}=p_{c}-p_{b}^{2} /\left(4 p_{a}\right)$. Based on this formulation, parcels where

$$
\begin{cases}p_{b}>0, t_{\text {min }}<0 & \rightarrow \text { moving far away from each other; } \\ d_{\text {min }}^{2}>\left(R O I_{S}+R O I_{L}\right)^{2} & \rightarrow \text { never close enough to collide; } \\ t_{\text {min }} \geq \Delta t & \rightarrow \text { too far to collide during current time-step. }\end{cases}
$$

This a priori selection significantly reduces the search space for potential collision partners, which otherwise would have to look at all possible couples, with $O\left(n_{p}^{2} / 2\right)$ computational demand.

\subsection{1. kd-tree based ROI collision estimation}

A pre-screening of potential candidate collision partners was developed using a kd-tree structured search algorithm. The kd-tree structure provides a sorted, binary partitioning of a dataset that allows for fast searches with a computational $\operatorname{cost} O(\log n)$ (Bentley (1975)). The kd-tree is defined recursively at the nodes. Each node contains a subset of the whole space, which is defined by dimensional bounds for each dimension, and has two children nodes. The subset is 
split between the children at the median value of its largest variance dimension, so that both branches departing from any node more or less contain the same number of elements. The tree is built starting from a root node, that contains the whole dataset, by recursively splitting each tree branch. Final 'leaf' nodes are reached whenever the dimensional span, or the number of items contained in a node, is small enough.

During this pre-processing phase, all possible candidates for which the conditions of Equation 31 are evaluated are mapped. The kd-tree structure is then used to perform nearest-neighbor searches that match the following, approximate rule:

$$
d\left(\mathbf{x}_{L 0}, \mathbf{x}_{S 0}\right) \leq R O I_{L}+\Delta t \boldsymbol{\theta}_{L},
$$

i.e., only the parcels which fall within the search sphere defined by the current $\mathbf{x}_{L}$ parcel position, having radius equal to $R O I_{L}$ plus the distance traveled by the parcel during the current time step, are filtered, as shown in Figure 5. The kd-tree structure has no effects on the accuracy of the search, but only serves the purpose of speeding up the evaluation of Equation 32 from $O\left(n^{2}\right)$ to $O(\log (n))$. This procedure introduces an approximation in the collision estimation model; however, at practical time-steps no noticeable differences could be observed, as reported in Figure 6 for a non-vaporizing spray simulation where $\Delta t=1 \mu \mathrm{s}$ was used. Figure 6 also shows that the computational time saved by using the kd-tree search procedure allowed a simulation speed-up of about one order of magnitude in comparison to the pure filtering with Equation 31 for a simulation with 20000 injected parcels. The standard binary collision search algorithm, where no ROIbased domain filtering is applied, needed a significantly larger simulation time, as reported in Figure 6. Even larger savings are seen for complex multi-injector simulations that can have hundreds of thousands of parcels.

When a collision is determined to occur, its outcome is computed based on the deterministic collision parameters. The collision impact parameter $b$ is given as the sine of the angle formed by the relative velocity vector and the line 


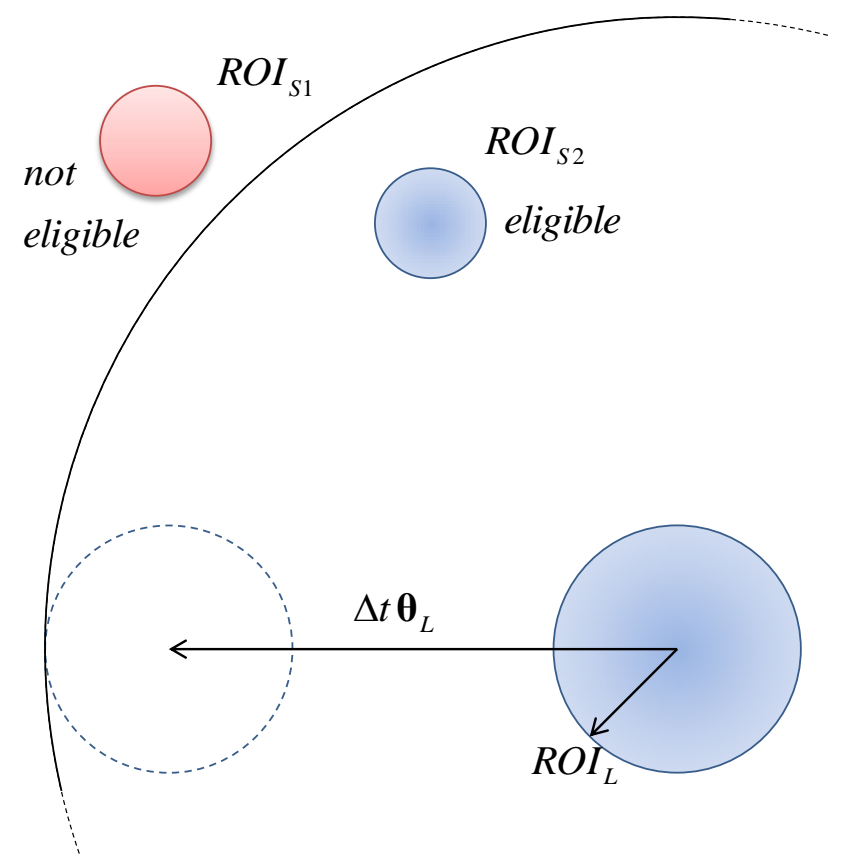

Figure 5: Pre-processing filter of candidate collision partners to parcel $L$.
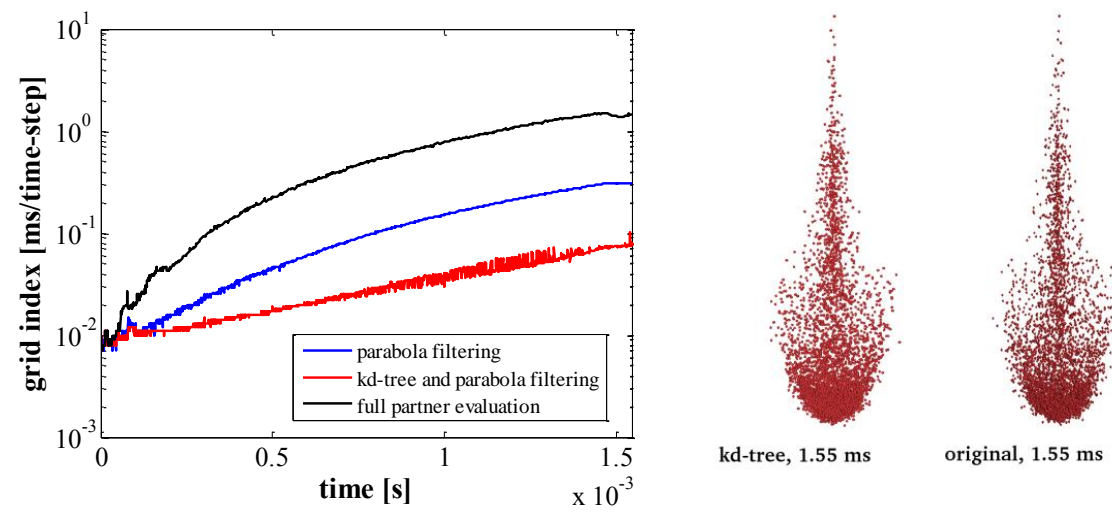

Figure 6: Collision eligibility method comparison: purely analytical estimation vs. analytical with kd-tree ROI search on a non-vaporizing, no breakup Spray A simulation with 20000 injected parcels. (left) CPU time spent per time-step; (right) view of the spray jets at $t=$ $1.55 \mathrm{~ms}$. 
connecting the droplet centers at time of impact, evaluated at the beginning of $W e_{c}$, drop diameter ratio $\Delta$ are also defined as:

$$
\begin{aligned}
b & =\sin \beta=\sqrt{1-\frac{\left\langle\boldsymbol{\theta}_{S}-\boldsymbol{\theta}_{L}, \mathbf{x}_{L 0}-\mathbf{x}_{S 0}\right\rangle}{\left\|\boldsymbol{\theta}_{S}-\boldsymbol{\theta}_{L}\right\|^{2}\left\|\mathbf{x}_{S 0}-\mathbf{x}_{L 0}\right\|^{2}}} ; \\
W e_{c} & =\frac{\rho_{S} r_{S}^{3}+\rho_{L} r_{L}^{3}}{r_{S}^{3}+r_{L}^{3}}\left\|\boldsymbol{\theta}_{S}-\boldsymbol{\theta}_{L}\right\|^{2} \frac{r_{S}}{\sigma} ; \\
\Delta & =r_{S} / r_{L} .
\end{aligned}
$$

Potential collision outcomes are computed following Munnannur and Reitz (2009), and feature 1) coalescence of the two parcels into a unique droplet, 2) 'grazing', or 'stretching separation' of parcels that only partially deviate their motion after the impact, 3) 'reflexive separation' where the parcels maintain their velocity after the impact, 4) pure bouncing where no mass is exchanged between the droplets. Fragmentation effects including formation of satellites are included as well. A comparison of the impact parameter-Weber diagram is reported in Figure 7 for a Spray A simulation with ethanol fuel, versus a set of measured collision outcomes for the same fuel and in ambient conditions in the experiments by Estrade et al. (1999).

\section{Sub-grid scale unsteady near-nozzle flow modeling}

In order to improve the accuracy of spray simulations in complex engine geometries, where the spatial resolution of the finite volume grid cells are typically coarser than the injector diameter, a robust unsteady gas-jet flow superimposition approach was developed and implemented. The approach assumes that close enough to the injector nozzle particle dynamics and their momentum coupling with the CFD gas-phase can be computed more accurately based on sub-grid scale velocity field predictions provided by a turbulent gas-jet model. 320 This allows use of meshes with resolution of up to few millimeters (Abani et al. (2008b,a); Wang et al. (2010)). 

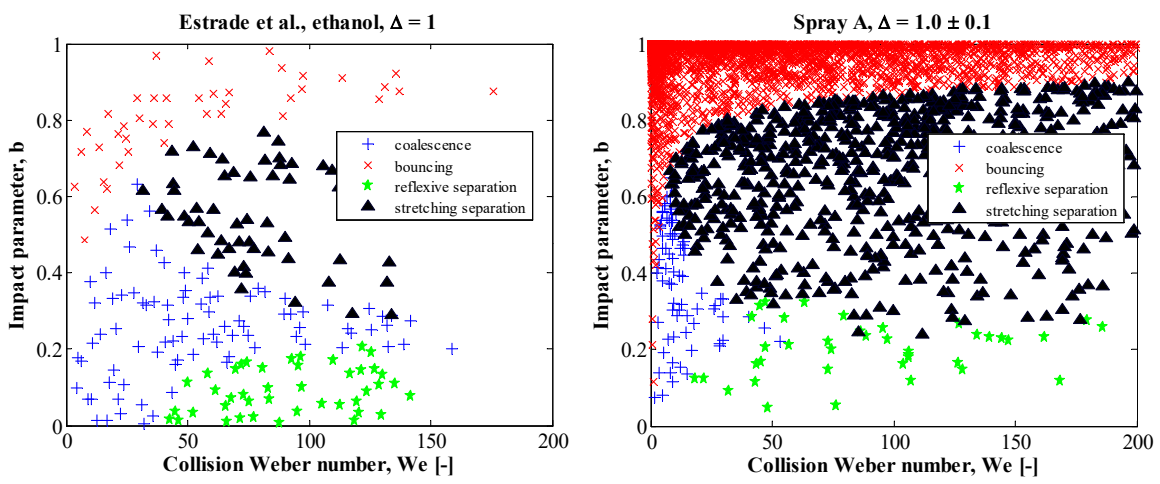

Figure 7: Comparison between measured (Estrade et al. (1999)) ethanol droplet collisions and predicted collisions in a non-vaporizing Spray A simulation with ethanol fuel using the current model.

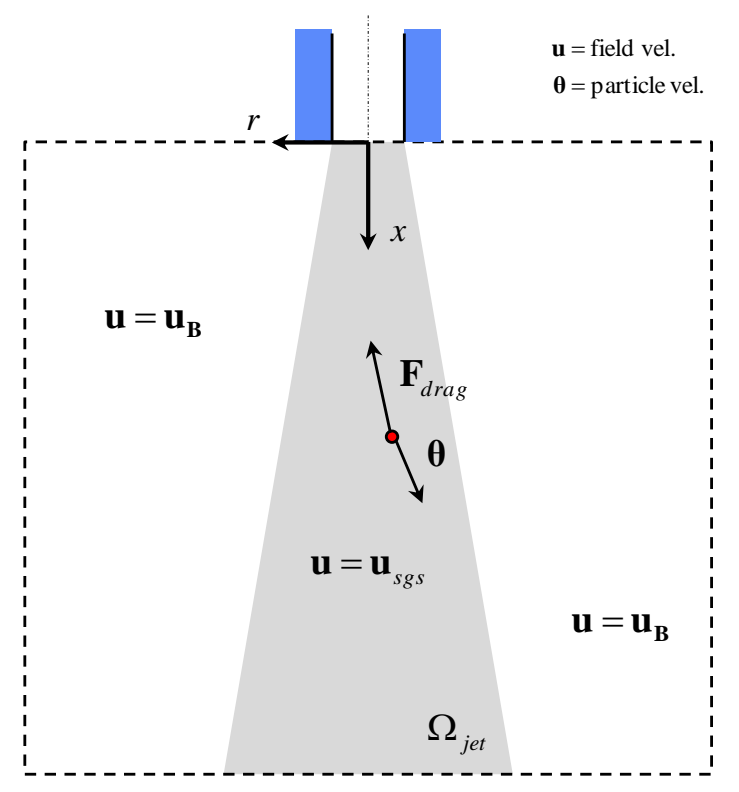

Figure 8: Schematic of application rule for sub-grid scale near-nozzle velocity field estimation. 
The unsteady gas-jet field model of Abani and Reitz (2007) was used, as reported in Figure 8. The implementation is based on the assumption that the two-phase spray jet is equivalent to a transient turbulent gas-phase jet having the same injection momentum and mass. The local flow gas-phase jet velocity $\mathbf{u}_{\text {axis }}(x, t)$ at a given time and position along the injection axis represents the convolution of $n$ successive velocity changes in the injection velocity $\mathbf{u}_{i n j}(t)$, from the start of injection $t_{0}$ :

$$
\begin{aligned}
\mathbf{u}_{a x i s}(x, t) & =\mathbf{u}_{i n j}\left(t_{0}\right)+\sum_{k=1}^{n}\left(1-\exp \left(-\frac{t-t_{k}}{\tau\left(x, t_{k}\right)}\right)\right)\left(\mathbf{u}_{i n j}\left(t_{k}\right)-\mathbf{u}_{i n j}\left(t_{k-1}\right)\right) ; \\
\tau(x, t) & =S t \frac{x-x_{i n j}}{\left|\mathbf{u}_{i n j}(t)\right|}
\end{aligned}
$$

330 Here, the jet response time $\tau\left(\mathbf{x}, t_{k}\right)$ is computed with the spray jet analogy, using the formulation of Crowe et al. (1997). The particle Stokes number, St, is a model calibration constant, assumed to be equal to a Strouhal number of the turbulent gas jet:

$$
\frac{f_{v} x}{u}=\text { Strouhal } \approx \text { Stokes }=\frac{\tau_{I}}{\tau_{p}},
$$

where $\tau_{I}$ represents the integral turbulence time scale, and $\tau_{p}$ is the particle 335 response time.

According to Abraham (1996), the effective injection velocity at the jet axis follows a logarithmic decay that starts from the jet downstream axial location $x_{0}=3 d_{e q} / K_{e n t r}$, where $d_{e q}=d_{n o z} \sqrt{\rho_{l} / \rho_{g}}$ is an effective gas jet diameter and $K_{\text {entr }}$ is a turbulent entrainment constant:

$$
\begin{array}{r}
\mathbf{u}_{a x i s, e f f}(x, t)=f(x) \mathbf{u}_{a x i s}(x, t), \\
f(x)=\frac{x_{0}}{x}, \forall x \geq x_{0} ;
\end{array}
$$

340 As the model does not provide a value for the viscous damping function $f(x)$ upstream of $x_{0}$, Abani and Reitz (2007) assumed a constant unity value, i.e., 
no damping applied in proximity of the injection point. Wang et al. (2010) reported however that using the predicted gas-jet axis velocity leads to increased average particle sizes, and proposed a smoothing function to reduce the effective predicted injection velocity upstream of $x_{0}$. A similar approach was adopted in this work. However, a more flexible and efficient algebraic formulation was developed where the effective injection velocity damping at the axial location $x$ is defined in terms of a normalized coordinate, $\chi=x / x_{0}$ :

$$
f(\chi)= \begin{cases}1 / \chi, & \chi \geq \varphi \\ \gamma_{\max }-k(\chi-1)^{2}, & 1 \leq \chi<\varphi \\ \gamma_{\min }+\chi(2-\chi)\left(\gamma_{\max }-\gamma_{\min }\right), & \chi<1\end{cases}
$$

where the function is parameterized to be of class $C^{2}$ within the whole domain, thus:

$$
\begin{aligned}
\Delta & =\sqrt{9-8 \gamma_{\max }} ; \\
k & =\frac{32 \gamma_{\max }^{3}}{(3+\Delta)^{2}\left(3+\Delta-4 \gamma_{\max }\right)} \\
\varphi & =\frac{3+\Delta}{4 \gamma_{\max }} .
\end{aligned}
$$

The independent parameters $\gamma_{\max }$ and $\gamma_{\min }$ are reported in Figure 9. Once the time-resolved, transient effective jet velocity at the spray axis is determined, the value at a radial location $r$ from the axis is provided by (Abraham (1996)):

$$
\mathbf{u}_{s g s}(x, r, t)=\frac{\mathbf{u}_{a x i s, e f f}(x, t)}{\left(1+\frac{12 r^{2}}{K_{e n t r}^{2} x^{2}}\right)^{2}} .
$$

Finally, one must define the domain $\Omega_{\text {sgs }}$ over which the unsteady sub-grid scale gas jet model is applied. We use a conic region whose angle is $10 \%$ wider than the injection cone angle, and an axial depth equal to twice the liquid breakup length of Levich (1962). 

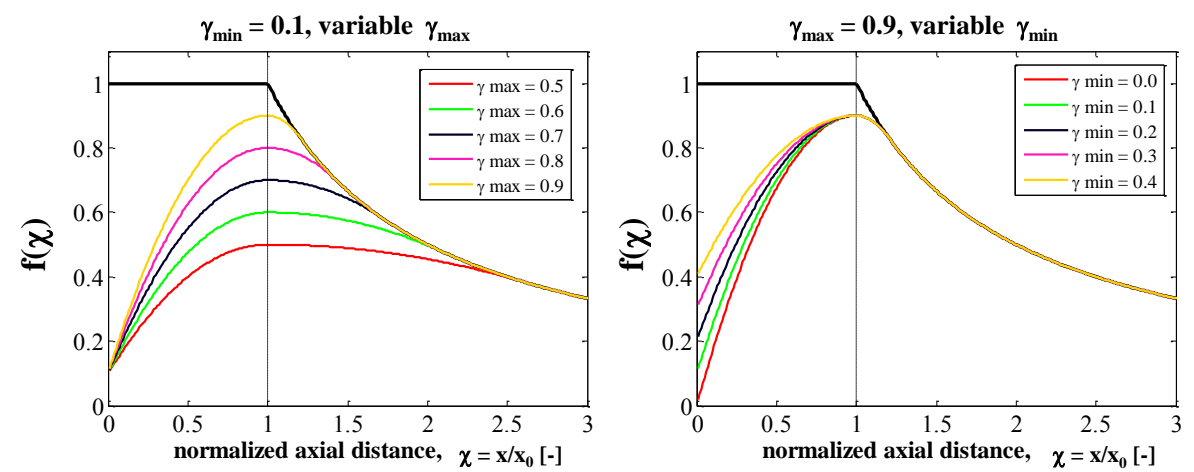

Figure 9: Effects of different parameter settings $\left(\gamma_{\min }, \gamma \max \right)$ for the axial velocity damping function, $f(\chi)$ of equation 41. Thick black line represents Abani and Reitz (2007).

\subsection{Particle momentum coupling solution with sub-grid scale flow}

The above models were applied within the KIVA code (Torres and Trujillo (2006)), which solves the mass conservation, momentum and energy conservation equations for weakly compressible, turbulent gas-phase flows with sprays. In the code, different strategies of operator splitting are applied to each equation, according to the Arbitrary Lagrangian-Eulerian (ALE) scheme of Butler et al. (1979). Particle transport is computed together with the flow momentum equation:

$$
\frac{D}{D t} \int_{\Omega} \rho \mathbf{u} d V=-\int_{\partial \Omega}\left(p+\frac{2}{3} \rho k\right) d \mathbf{A}+\int_{\partial \Omega} \boldsymbol{\sigma} \cdot d \mathbf{A}+\int_{\Omega} \mathbf{F}_{p} d V
$$

where $\mathbf{F}_{p}$ represents particle-related momentum coupling terms. Discretization of Equation 46 happens during the Lagrangian step ('time $n$ ' to 'time $B$ '), which is computed on a locally moving grid, and does not consider advective transport, taking the following form:

$$
\begin{cases}m_{B} \mathbf{u}_{B}-m_{n} \mathbf{u}_{n} & =\mathbf{E}-\sum_{p \in \Omega} \frac{4}{3} \pi \rho_{p} N_{p}\left[r_{p, B}^{3} \boldsymbol{\theta}_{B}-r_{p, n}^{3} \boldsymbol{\theta}_{n}^{\prime}\right] \\ \boldsymbol{\theta}_{B}-\boldsymbol{\theta}_{n}^{\prime} & =\Delta t\left[d_{p}\left(\mathbf{u}_{B}+\boldsymbol{\theta}_{n}^{t}-\boldsymbol{\theta}_{B}\right)\right]\end{cases}
$$

where $\mathbf{E}$ represents all non-spray related terms. $\boldsymbol{\theta}_{n}^{\prime}$ represents an already partially updated parcel state where breakup and collisions have been computed, 
and $\boldsymbol{\theta}_{n}^{t}$ represents turbulent dispersion velocity. If the particle drag coefficient of Equation 14, is assumed to be constant within the integration step, $d_{p}=d_{p, n}$ and the two systems of equations become linearly dependent. Thus, the field equations can be solved before actually computing the updated particle velocities:

$$
\left(m_{B}+S_{u v w}\right) \mathbf{u}_{B}-m_{n} \mathbf{u}_{n}=\mathbf{E}-\mathbf{r}_{\mathbf{u}},
$$

where $S_{u v w}$ and $\mathbf{r}_{\mathbf{u}}$ are constant mass and momentum coupling terms during the iterations:

$$
\forall p \notin \Omega_{s g s},\left\{\begin{array}{l}
S_{u v w}=\frac{4}{3} \pi \rho_{p} N_{p} r_{B}^{3} \frac{\Delta t d_{p}}{1+\Delta t d_{p}}, \\
\mathbf{r}_{\mathbf{u}}=\frac{4}{3} \pi \rho_{p} N_{p}\left(r_{B}^{3} \frac{\boldsymbol{\theta}_{n}^{\prime}+\Delta t d_{p} \boldsymbol{\theta}_{t}}{1+\Delta t d_{p}}-r_{n}^{3} \boldsymbol{\theta}_{n}^{\prime}\right) .
\end{array}\right.
$$

As seen in Figure 8, the second of Equation 47 does not depend on computed

380 values of the velocity field:

$$
\boldsymbol{\theta}_{B}-\boldsymbol{\theta}_{n}^{\prime}=\Delta t\left[d_{p}\left(\mathbf{u}_{s g s}+\boldsymbol{\theta}_{n}^{t}-\boldsymbol{\theta}_{B}\right)\right],
$$

thus:

$$
\forall p \in \Omega_{s g s},\left\{\begin{array}{l}
S_{u v w}=0 \\
\mathbf{r}_{\mathbf{u}}=\frac{4}{3} \pi \rho_{p} N_{p}\left(r_{B}^{3} \frac{\boldsymbol{\theta}_{n}^{\prime}+\Delta t d_{p}\left(\boldsymbol{\theta}_{t}+\mathbf{u}_{s g s}\right)}{1+\Delta t d_{p}}-r_{n}^{3} \boldsymbol{\theta}_{n}^{\prime}\right)
\end{array}\right.
$$

\section{Genetic-algorithm-based study of model constants}

The introduction of the near-nozzle sub-grid scale flow model adds further model constants to spray simulations and also modifies the performance of other models, affecting breakup predictions. Because of the large number of constants, a genetic algorithm-based study was set up to find an optimal set that best matches transient spray data. This study also helps understand the sensitivity of the model to each constant and the near-nozzle entrainment profile parameters. 
The Engine Combustion Network Spray A experiments by Pickett et al. (2011) were chosen as they have also been seen to be repeatable across different institutions (Meijer et al. (2012)).

The multi-objective optimization used the NSGA-II algorithm of Deb et al. (2002), with the five following merit functions, as also summarized in Figure 10:

$$
\begin{gathered}
f_{1}=\bar{v}=\frac{1}{t} \int_{0}^{t}\left(\frac{v_{\text {sim }}(\tau)-v_{\text {exp }}(\tau)}{v_{\text {exp }}(\tau)}\right)^{2} d \tau \\
f_{2}=\frac{1}{t_{\text {ramp }}} \int_{0}^{t_{\text {ramp }}}\left(\frac{l_{\text {sim }}(\tau)-l_{\text {exp }}(\tau)}{l_{\text {exp }}(\tau)}\right)^{2} d \tau \\
f_{3}=\frac{\left|\bar{l}_{\text {sim }, q s s}-\bar{l}_{\text {exp }, q s s}\right|}{\bar{l}_{\text {exp }, q s s}}, \\
f_{4}=\frac{\left|\sigma\left(l_{\text {sim }, q s s}\right)-\sigma\left(l_{\text {exp }, q s s}\right)\right|}{\sigma\left(l_{\text {exp }, q s s}\right)}, \\
\left.\varepsilon_{z}\left(y_{f}\right)\right|_{z_{0}}=\int_{0}^{r_{\max }} \int_{0}^{2 \pi}\left(\frac{y_{f, s i m}\left(r, \phi, z_{0}\right)-y_{f, e x p}\left(r, \phi, z_{0}\right)}{y_{f, e x p}\left(r, \phi, z_{0}\right)}\right)^{2} d \phi d r \\
\varepsilon_{a x}\left(y_{f}\right)=\int_{z_{i n j}}^{z_{\max }}\left(\frac{y_{f, s i m}(0,0, z)-y_{f, e x p}(0,0, z)}{y_{f, \exp }(0,0, z)}\right)^{2} d z, \\
f_{5}=\left.\varepsilon_{z}\left(y_{f}\right)\right|_{2 c m}+\left.\varepsilon_{z}\left(y_{f}\right)\right|_{3 c m}+\left.\varepsilon_{z}\left(y_{f}\right)\right|_{4 c m}+\left.\varepsilon_{z}\left(y_{f}\right)\right|_{5 c m}+\varepsilon_{a x}\left(y_{f}\right) .
\end{gathered}
$$

The first objective, $f_{1}$, represents how well the simulation captures the global vapor phase penetration $v$, and is computed as its mean squared error versus the experimental datum. The second objective, $f_{2}$, monitors the simulation accuracy in predicting the initial injection transient. The mean squared error of liquid penetration $l$ is also computed. The following two objectives, $f_{3}, f_{4}$, target the absolute value and the stability of the liquid-phase penetration in the nearly steady-state phase of the injection event, by monitoring the relative error of the time-averaged predicted vs. experimental liquid penetration and of its standard deviation, respectively. Finally, the last objective considers local mixture fraction predictions. Using ensemble-averaged imaging from the Spray A data, radial and axial mixture fraction profiles were obtained as reported 
in Figure 11: four different axial locations at $d z=20,30,40,50 \mathrm{~mm}$ from the injector nozzle are used to provide the mixture fractions. The data were collapsed into a single objective function, which represents the sum of the integral root mean squared error of the mixture fraction predictions of all 5 observation planes, as reported in eqs. (56) to (58).
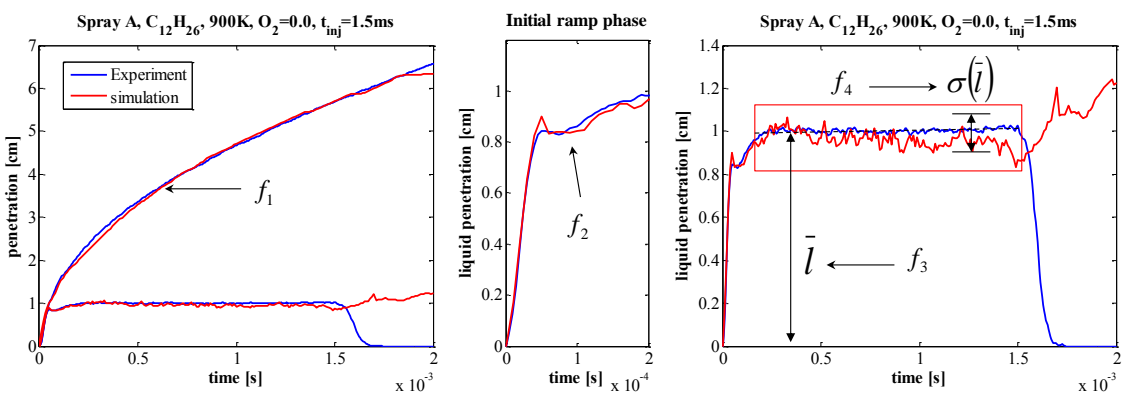

Figure 10: Schematic of Spray A penetration measurements used in the multi-objective optimization: $\left(f_{1}\right)$ integral vapor penetration, $\left(f_{2}\right)$ liquid penetration in the initial transient $(t \leq 100 \mu s),\left(f_{3}, f_{4}\right)$ liquid penetration in the quasi-steady-state phase $(300 \mu s \leq t \leq 1.5 \mathrm{~ms})$.
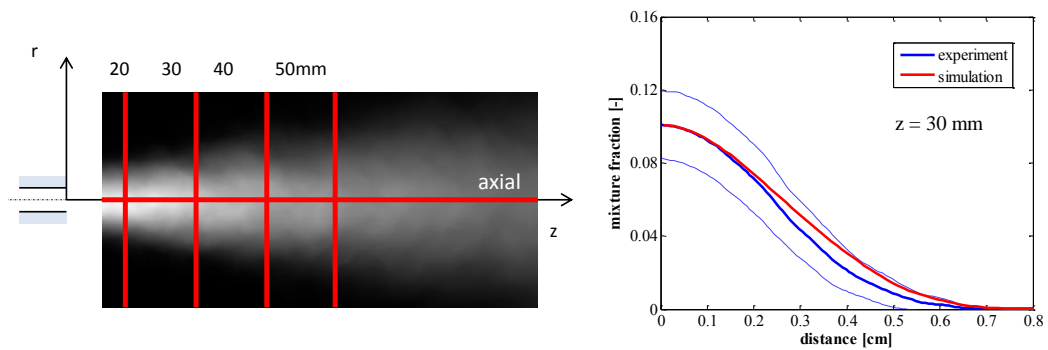

Figure 11: Schematic of Spray A mixture fraction distributions used in the multi-objective optimization: $\left(f_{5}\right)$ radial distribution at $t=1.45 \mathrm{~ms}$ and $d z=20,30,40,50 \mathrm{~mm}$ from the nozzle; axial distribution at $t=1.45 \mathrm{~ms}$.

Selection of the model constants to be optimized was constrained to the six parameters reported in Table 1, while the remaining non-optimized model parameters were selected from literature data.

415 The genetic optimization was run using a population of 200 individuals, and 


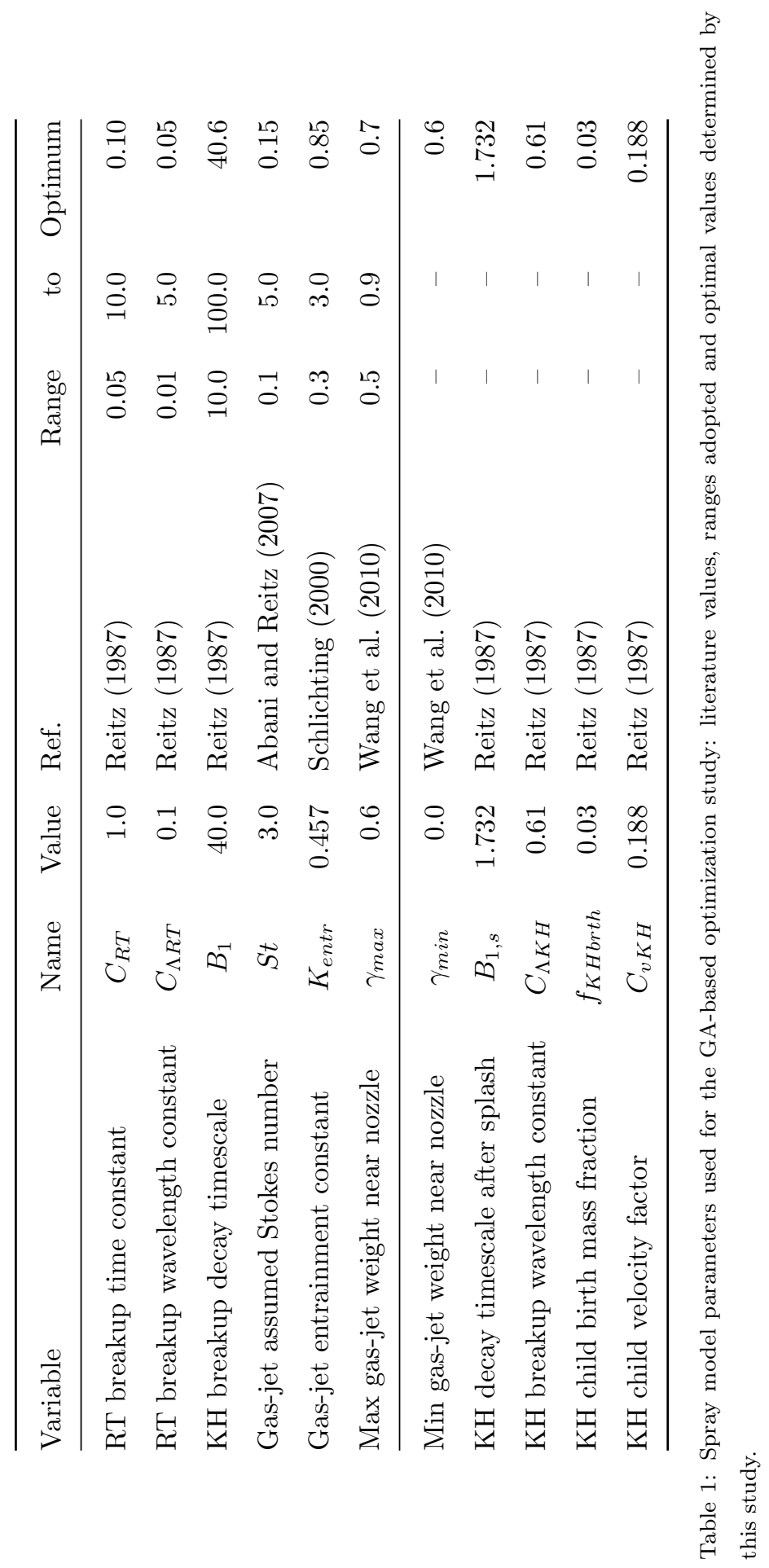


evolved for 100 successive generations, for a total of 20000 runs. Optimal individuals have the lowest values of the selected fitness functions. A compact representation of the optimization output is reported in Figure 12, where each plot shows the correlation between the i-th (row) merit function and the j-th ${ }_{420}$ (column) variable values for all individuals in the optimization. All points are colored by ranges of mixture fraction distribution merit, which was regarded as the most important parameter for the application of the spray model for practical combustion simulations.

A noticeable correlation in the first two merit functions is highlighted by the first two rows in the plot matrix, showing very similar behavior as far as the sub-grid scale model constants are concerned. Global vapor penetration is as accurate as the initial liquid-phase ramp up transient, indicating the importance of modeling the initial injection transient for overall simulation accuracy. Furthermore, both $f_{1}$ and $f_{2}$ show an almost linear correlation with the assumed Stokes number in the gas-jet analogy: lower values of $S t$ represent more responsive jets, which also leads to earlier liquid phase development and faster vapor tip penetration. A responsive near-nozzle gas-jet representation is necessary to capture spray and vapor phase development starting from the beginning of the injection, when the local (under-resolved) CFD prediction has not yet correctly 435 developed. A similar trend was observed in the entrainment constant $K_{\text {entr }}$, which controls the velocity decay profile within the jet, both axially and radially, hence affecting spray tip penetration. The genetic optimization converged to a value of $K_{\text {entr }} \approx 0.85$, which is slightly larger than the value of 0.7 proposed by Abani and Reitz (2007). For the near-nozzle sub-grid scale model smoothing parameter $\gamma_{\max }$, a well-defined Pareto front was reached, at $\gamma_{\max }=0.7$.

The breakup model constants affected the simulation results in a less straightforward way. The KH timescale constant converged to $B_{1}=40.6$ for accurate vapor penetration, initial liquid ramp and mixture fraction distributions. Higher values of $B_{1}$, up to $B_{1} \approx 50$ appeared to be beneficial for capturing both liquid length value and its standard deviation. A lower value of the $\mathrm{KH}$ timescale con- 
stant reduces the particle's lifetime and speeds up momentum transfer to the gas phase. This competes with the larger jet dispersion of the RNG k- $\epsilon$ model (see Pope (1978)). The effect of the RT model constants is reported in Figure 13 , which shows the best $15 \%$ of individuals with respect to merit functions $f_{1}$ (vapor tip penetration) and $f_{5}$ (mixture fraction distribution). The RT wavelength constant affects especially the gas-phase predictions, and small values are preferred. The drop size does not affect liquid penetration as much, and no relevant $C_{\Lambda R T}$ value patterns were found by the GA with respect to $f_{2}, f_{3}, f_{4}$ - Instead, the RT timescale constant $C_{\tau R T}$, significantly affects the standard deviation of the steady-state liquid length, suggesting that values of $C_{\tau R T} \leq 1.5$ should be used to avoid broad fluctuations of the liquid length. This is in line with the suggested unity value from Reitz (1987). However, the present GA predictions also exhibit trade-offs. For example, the vapor penetration and mixture fraction distribution objectives suggest an optimal RT timescale constant to be of the order of $C_{\tau R T} \approx 0.1$. Similarly to that observed for KH breakup, an optimal RT timescale constant for stable liquid length prediction of objective $f_{3}$ requires a value $C_{\tau R T}=2.39$, in contrast with what is needed for precise vapor phase modeling.

\section{Validation}

The genetic algorithm's optimal set of spray model constants are reported in the last column of Table 1 . Using this set the spray model was validated against experimental constant volume vessel sprays and mixture preparation in a light-duty optically accessible engine. The simulations were conducted using the KIVA CFD code (Torres and Trujillo (2006)), with the sub-models reported in Table 2.

\subsection{Engine Combustion Network Spray-A}

The Engine Combustion Network (ECN) Spray A experiment of Pickett 475 et al. (2011) features a single-pulse, long injection of n-dodecane fuel into a 


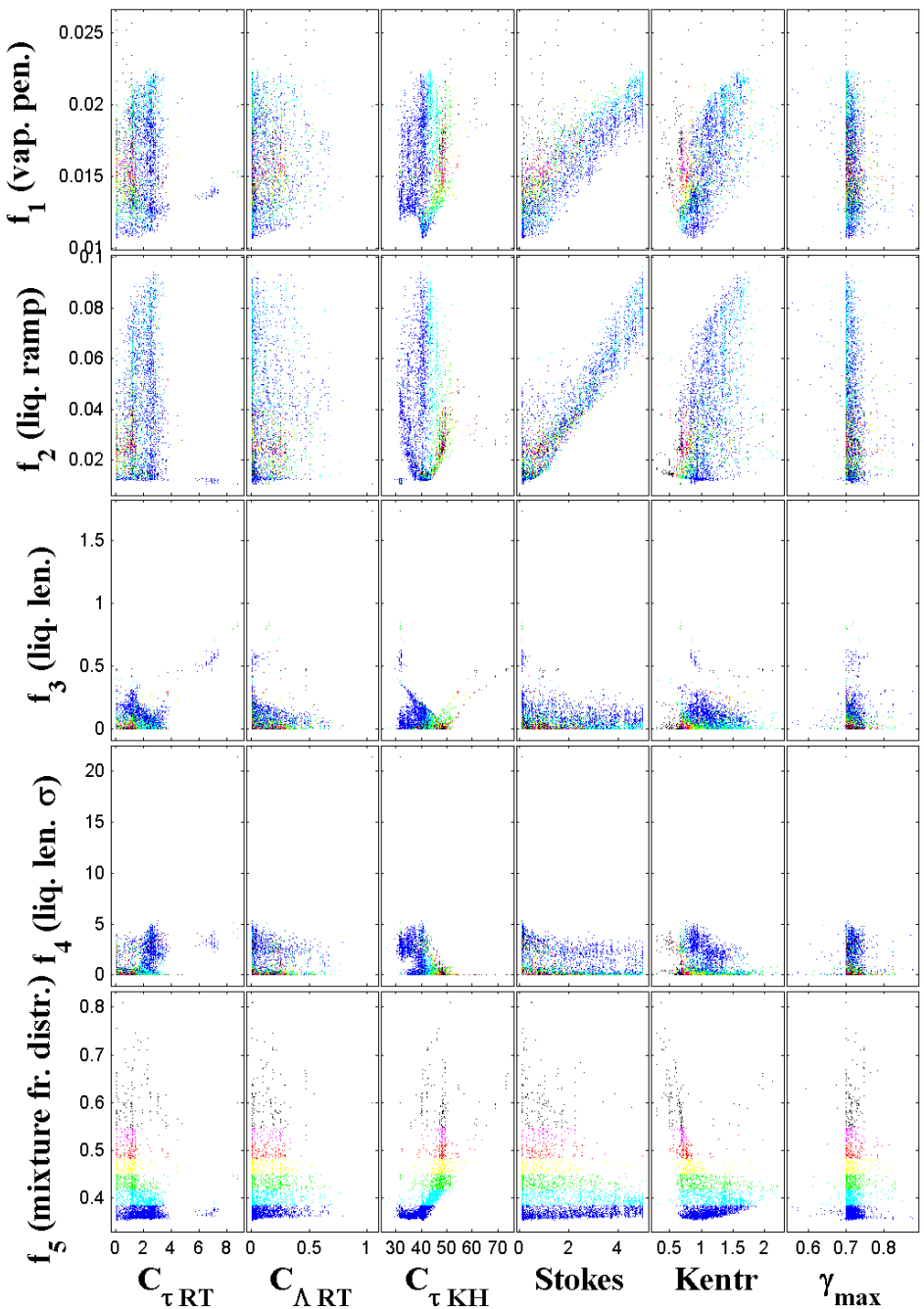

Figure 12: Pareto front splitting matrix of the multi-objective, multi-variate optimization. Each plot represents correlation between the i-th-row merit function and the j-th-column optimized variable. Colour by mixture fraction distribution merit range: (blue) best to (black) worst. 


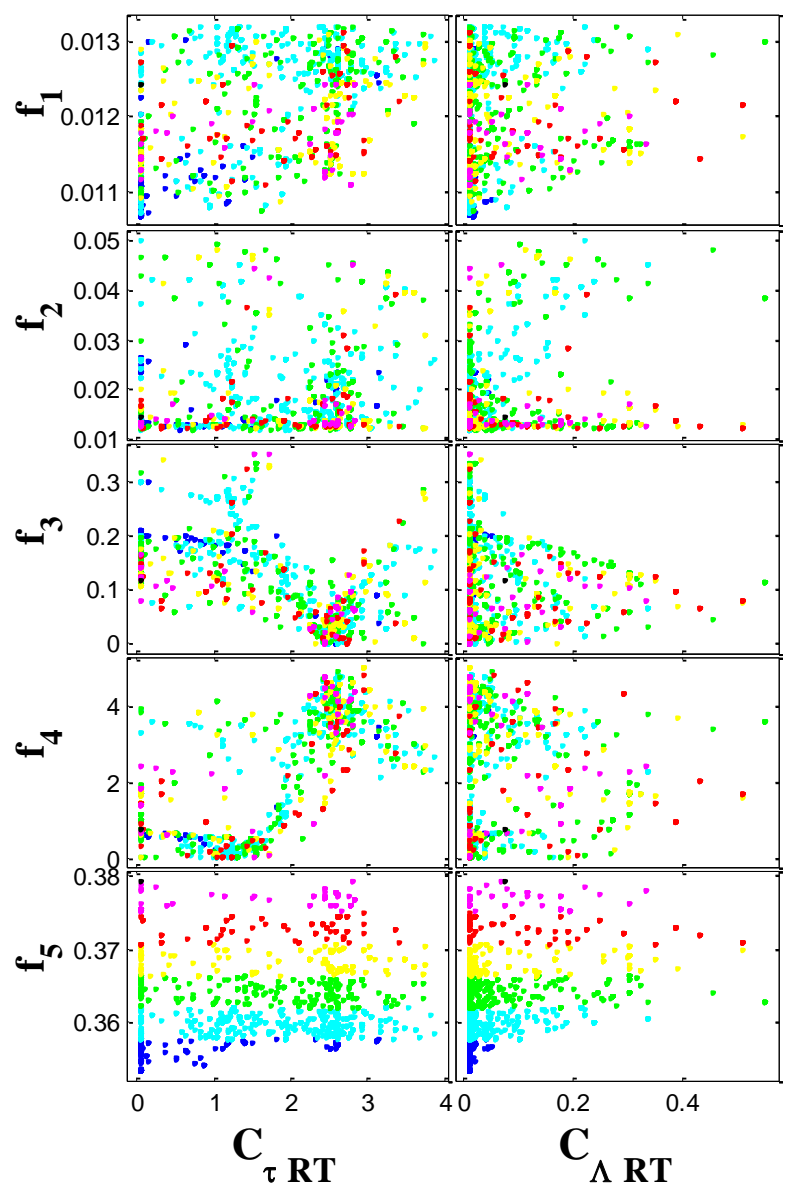

Figure 13: Pareto front splitting matrix of the optimized RT breakup parameters: $C_{\tau R T}$, $C_{\Lambda R T}$ versus corresponding merit function. Filtered subset representing $15 \%$ of the best individuals in $f_{1}$ (vapor penetration) and $f_{5}$ (mixture fraction distribution). 
high-pressure and high-temperature constant volume vessel. Corresponding simulations were run using the detailed initial and boundary conditions provided on the Sandia website (Sandia (2014)). A three-dimensional constant-volume mesh featuring 70224 cells was used, as shown in Figure 14. This mesh has an average cell resolution of $0.35 \times 0.35 \times 2.5 \mathrm{~mm}$ near the nozzle. The axial resolution coarsens following a geometric progression with ratio 1.04, while the radial resolution coarsens following a semi-cone angle of 3 degrees. A summary of the initial and boundary conditions for the experiment is reported in Table 3.

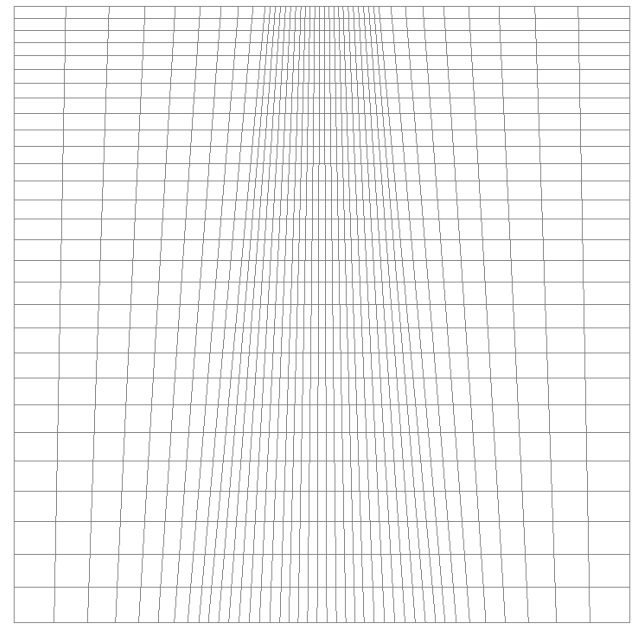

Figure 14: Vertical cross section of the computational grid used for Spray A simulations.

Figures 16 and 17 show the liquid and gas-phase penetration time histories versus measured values. The liquid and vapor penetrations are matched well, even though some underestimation of the gas-phase penetration is seen. Figure 18 shows that the model captures the mixture fraction distribution well at all four axial locations, and especially the global entrainment (maximum jet radial coordinate) is well optimized. 


\begin{tabular}{lll}
\hline Model & Name & Ref. \\
\hline Solver & ALE, SIMPLE & Amsden et al. (1989) \\
Turbulence & RNG k- $\epsilon$ & Han and Reitz (1995) \\
Vaporization & 1-D multi-component & Torres et al. (2003) \\
Law-of-the-wall & - & Launder and Spalding (1972) \\
\hline
\end{tabular}

Table 2: Sub-models activated in the flow solver for validation of the spray simulations in this study.

\begin{tabular}{ll}
\hline \multicolumn{2}{c}{ Vessel conditions } \\
\hline Composition & $\mathrm{N}_{2}: 0.8971$ \\
& $\mathrm{CO}_{2}: 0.0652$ \\
& $\mathrm{H}_{2} \mathrm{O}: 0.0377$ \\
Pressure & $60.45 \mathrm{bar}$ \\
Temperature & $900 \mathrm{~K}$ \\
Density & $22.8 \mathrm{~kg} / \mathrm{m}^{3}$ \\
\hline \multicolumn{1}{c}{ Injector specifications } \\
\hline Type & common-rail \\
Nozzle & single-hole \\
Nozzle diameter & $0.084 \mathrm{~mm}$ \\
Injection pressure & $150 \mathrm{MPa}$ \\
Injection duration & $1.5 \mathrm{~ms}, 6.0 \mathrm{~ms}$ \\
Fuel type & $n C_{12} \mathrm{H}_{26}$ \\
Fuel mass & $3.47 \mathrm{mg}, 13.77 \mathrm{mg}$ \\
\hline
\end{tabular}

Table 3: Summary of the 'Spray A' vessel and injection conditions (Pickett et al. (2011)). 
Some overestimation of centerline mixture fraction is seen in the closer planes to the injector: as reported in Pope (1978), k-epsilon models suffer from overestimating jet dispersion and underestimating penetration. While Pope (1978) suggested a tuning of the k-epsilon constants to overcome this issue, valid for gaseous jet simulations, this was not viable in this study, as the flow field conditions that foster combustion development in engines are mainly achieved through compression and swirling flows. The calibration found by the GA suggests a way to capture correct jet penetration and dispersion with a RNG k-epsilon model, even if at the price of accepting higher centerline mixture fractions very close to the liquid-gas phase transition.

Figure 15 shows the predicted initial liquid-phase development transient in the Spray A experiment by Manin et al. (2012). This comparison highlights the good accuracy of the calibrated model prediction in terms of both tip penetration and spray cone angle (the dashed lines represent measured liquid length data as reported by Pickett et al. (2011)). The experiment reveals a robust liquid core prior to RT breakup. The simulation predicts that the RT particles are located in the lighter cloud region, while the $\mathrm{KH}$ blobs match well the dense, darker-colored liquid bulk region.

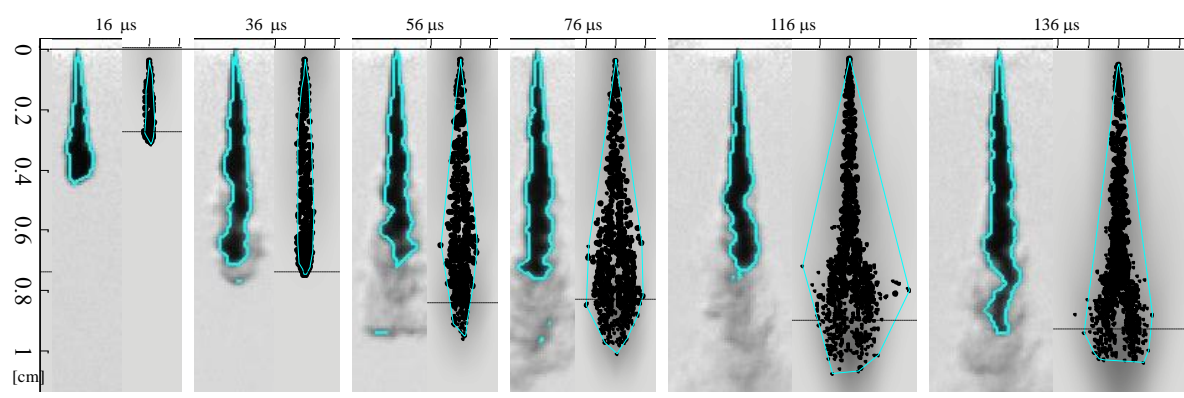

Figure 15: Validation of Spray A early liquid penetration. Diffused back illumination from Manin et al. (2012); (dashed lines): ensemble-averaged liquid penetration from Pickett et al. (2011). Parcel size proportional to computed drop size.

Figure 19 also shows the effects of selecting a different number of injected spray parcels on predicted liquid spray penetration for the Spray A. As the 


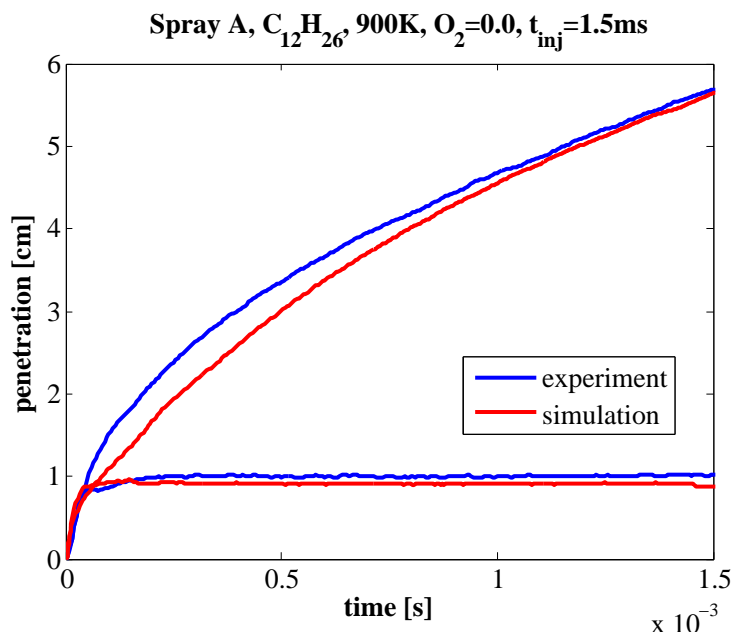

Figure 16: Validation of Spray A liquid and vapor penetration, $t_{i n j}=1.5 \mathrm{~ms}$. Experimental data from Pickett et al. (2011).

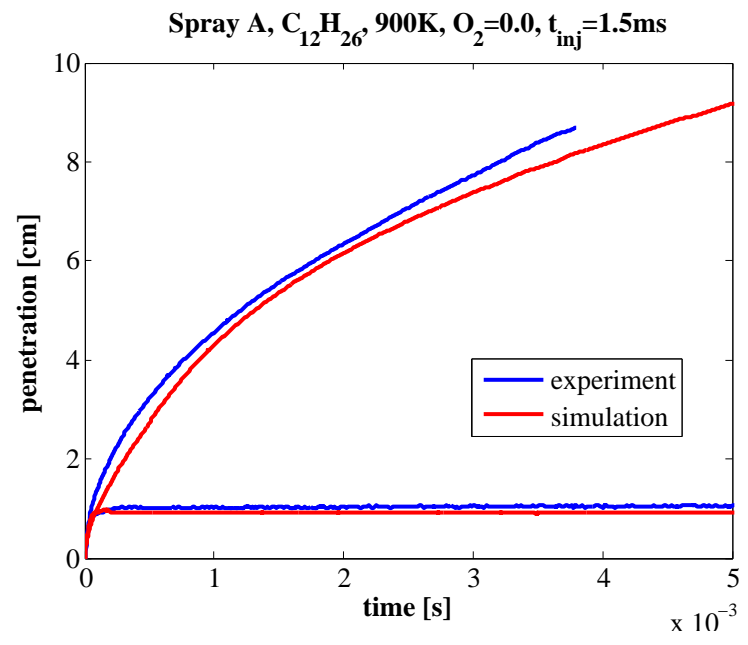

Figure 17: Validation of Spray A liquid and vapor penetration, $t_{i n j}=6.0 \mathrm{~ms}$. Experimental data from Pickett et al. (2011). 

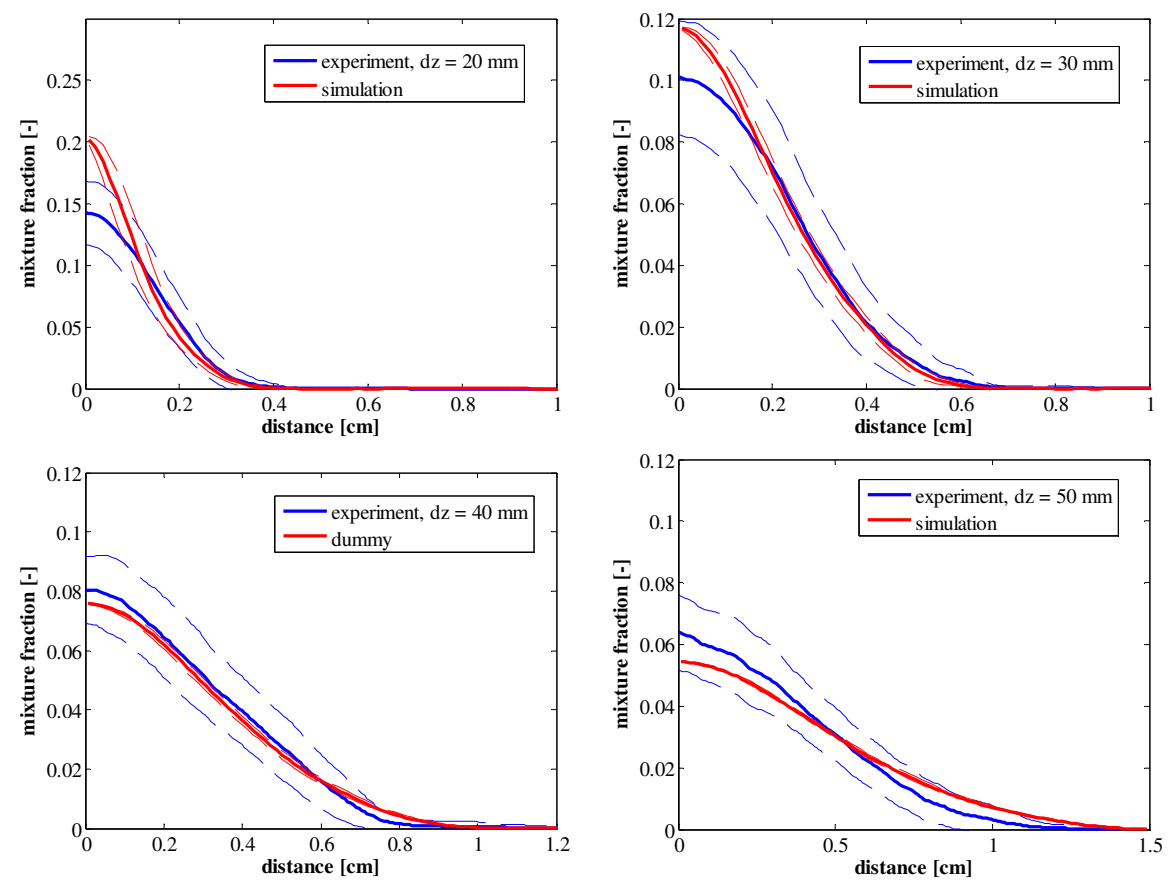

Figure 18: Mixture distribution comparison for Spray A case, at $t=4.77 m s$ (Pickett et al. (2011)). 


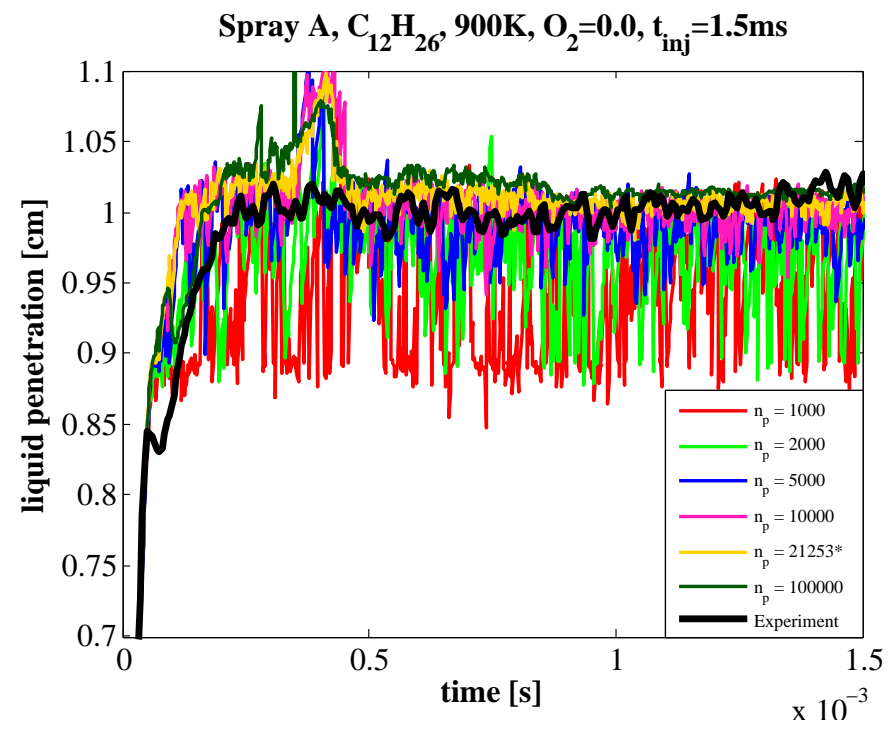

Figure 19: Effects of number of injected parcels and 'full' blob model injection on predicted Spray A liquid penetration.

figure shows, the 'full' blob injection model using Equation 2 is able to reduce the number of under-resolved parameters in the spray simulation by achieving a converged representation of the liquid spray. For the Spray A simulation, 21253 parcels are injected. All simulations with lower numbers of particles showed excessive variance in the liquid penetration curve, conveying that the number of discrete particles in the simulation was not large enough to represent the stable liquid penetration height. Instead, artificially increasing the number of parcels up to $100 \mathrm{k}$, where each injected parcel accounted for about 0.2 blobs, did not improve the simulation results, but showed a converged behavior.

\subsection{Mixture preparation in a light-duty optical diesel engine}

Partially-premixed combustion strategies in light-duty engines are characterized by a unique, late injection pulse which targets the bowl rim in order to split the fuel jet between the bowl and the squish regions, as an attempt to

${ }_{525}$ foster fuel-air mixing by increasing the available surface for air entrainment into the fuel jet (Musculus et al. (2013)). At the high levels of dilution operated 
in these cases, it was observed that significant equivalence ratio stratification is needed to ignite the fuel-air mixture with mildly-to-severely-rich pockets, and to propagate combustion to the overly lean mixture covering most of the combustion chamber. Also, overly lean mixture in the squish region apperared to be the responsible for most of the $\mathrm{CO}$ and unburnt hydrocarbon (UHC) emissions from these cases (Sahoo et al. (2011)).

Hence, spray modeling for such operating conditions needs to capture the local mixture distributions. The experimental study of mixture preparation in a GM light duty diesel engine by Sahoo et al. (2011) was adopted to further validate the spray model. Equivalence ratio measurements were made in the optically accessible diesel engine, derived from a current production four cylinder 1.9L engine. The engine was equipped with a fused-silica piston top which retained the full geometrical details of the metal piston, including valve recesses. A 540 Bosch CRIP2.2 injector was mounted vertically, aligned with the cylinder axis; the injector hole protrusion into the combustion chamber was $0.3 \mathrm{~mm}$ below the fire-deck. For simplicity, a computational mesh representing one seventh of the combustion chamber was used for the simulations, as represented in Figure 20. A full description of the experimental engine and injection system setup can be found in Sahoo et al. (2011), while a summary of the main details of the operating condition for the present study is reported in Table 4.

The experimental measurements were carried out using planar laser-induced fluorescence (PLIF) in a non-reacting nitrogen charge, whose initial conditions were set to match the intake flow rate and temperature at TDC of a reference combusting case's operating conditions. This reference condition features a high EGR ratio, corresponding to an intake oxygen molar fraction of $10 \%$, and a swirl ratio $R_{s}=2.20$. The fixed injected amount of a primary reference fuel, made up of $25 \%$ iso-octane and $75 \%$ n-heptane was used with three different injection pressures: $p_{i n j}=500,860,1220$ bar, with different injection rate laws and durations, as reported in Figure 21, measured by Busch (2014).

Comparisons between measured and experimental equivalence ratio images is reported in Figures 22,23,24. The comparisons were obtained at three dif- 


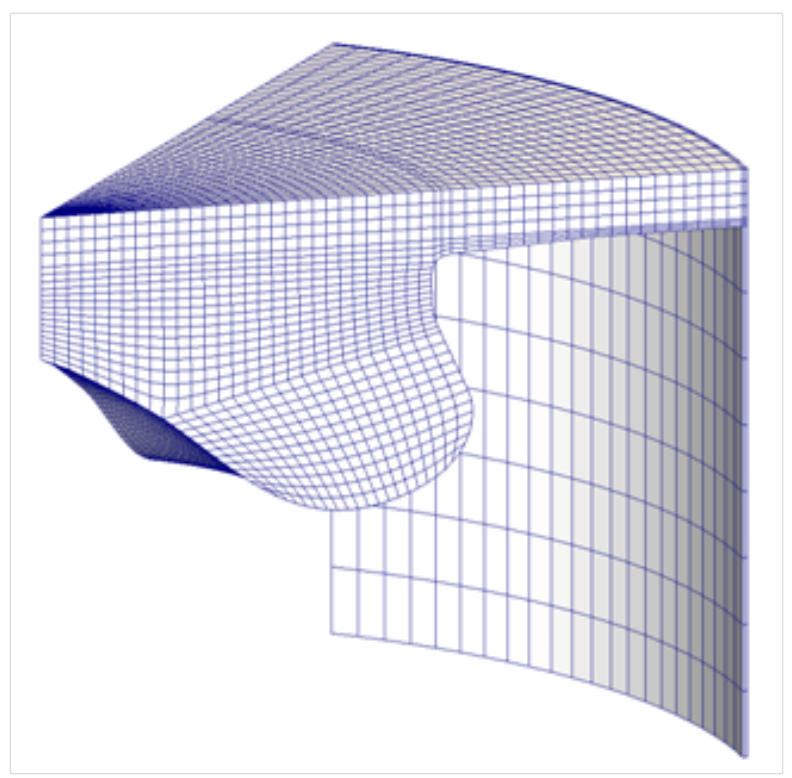

Figure 20: View of the computational grid used for mixture preparation study, near TDC.

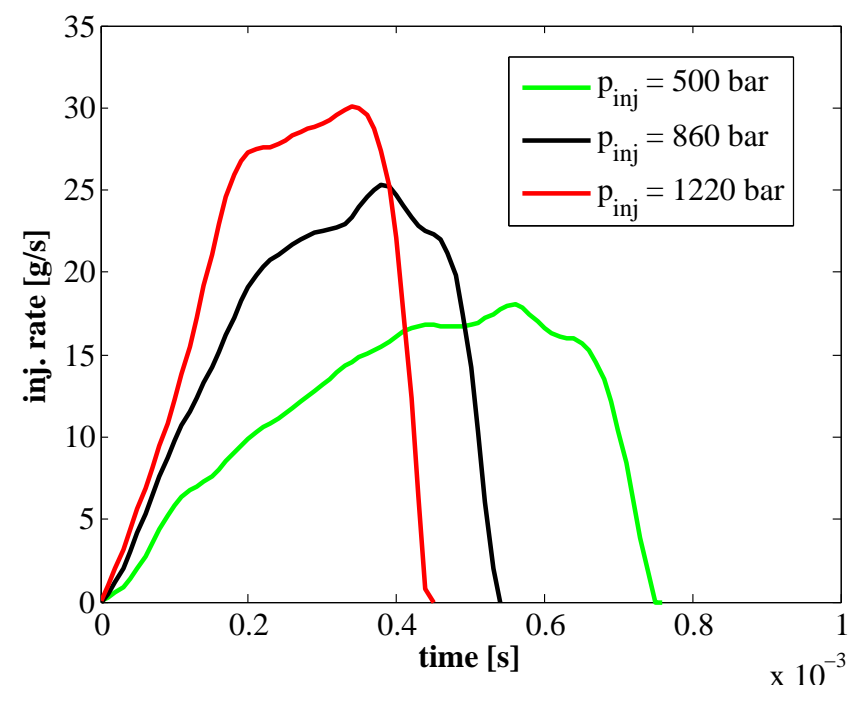

Figure 21: Injection rates for the $8.8 \mathrm{mg}$ injection pulse at 500,860,1220bar injection pressure (Busch (2014)). 


\begin{tabular}{|c|c|}
\hline \multicolumn{2}{|c|}{ Engine specifications } \\
\hline Bore $\times$ stroke $[\mathrm{mm}]$ & $82.0 \times 94.0$ \\
\hline Unit displacement $\left[\mathrm{cm}^{3}\right]$ & 477.2 \\
\hline Compression ratio & $16.4: 1$ \\
\hline Squish height at TDC $[\mathrm{mm}]$ & 0.88 \\
\hline \multicolumn{2}{|c|}{ Injector specifications } \\
\hline Type & Bosch CRIP 2.2 \\
\hline Sac volume $\left[\mathrm{mm}^{3}\right]$ & 0.23 \\
\hline Number of holes & 7 \\
\hline Included angle $[\mathrm{deg}]$ & 149.0 \\
\hline Hole diameter $[\mathrm{mm}]$ & 0.14 \\
\hline Hole protrusion $[\mathrm{mm}]$ & 0.3 \\
\hline \multicolumn{2}{|c|}{ Operating conditions } \\
\hline Charge composition & $100 \% N_{2}$ \\
\hline Intake pressure $[$ bar $]$ & 1.50 \\
\hline Intake temperature $[K]$ & 300 \\
\hline Engine speed $[\mathrm{rev} / \mathrm{min}]$ & 1500 \\
\hline \multicolumn{2}{|c|}{ Injection properties } \\
\hline Fuel type & $25 \% i C_{8} H_{18}, 75 \% n C_{7} H_{16}$ (vol.) \\
\hline Equivalent Cetane Number & 47 \\
\hline Injected fuel mass $[g]$ & 0.0088 \\
\hline Start of Injection $[\mathrm{deg}]$ & $-23.3 \pm 0.1$ \\
\hline Injection pressure $[\mathrm{MPa}]$ & $50.0,86.0,122.0$ \\
\hline
\end{tabular}

Table 4: Summary of the engine operating conditions for the three mixture preparation simulations (Perini et al. (2013)). 
ferent horizontal plane positions in the combustion chamber: approximately bisecting the squish volume height; at the piston bowl rim edge; and deep into the piston bowl volume, at its maximum radius position. Three crank angles were compared: $C A=[-15.0,-10.0,-5.0]$ degrees aTDC. The lowest injection pressure, $p_{i n j}=500 b a r$ (Figure 22) is also the farthest from the Spray A conditions $\left(p_{i n j}=1500 b a r\right)$. The spray jet penetration into the squish region is well captured for all three crank angles, and almost no jet dispersion is seen towards the central part of the combustion chamber. The same applies in the bowl rim plane, where lower equivalence ratios are seen. Penetration into the bowl plane, after hitting the bowl rim and traveling along the piston bowl wall is reasonably well captured. The simulation does a better job at capturing mixture preparation at higher injection pressures. At $p_{i n j}=860 \mathrm{bar}$, Figure 23 , the jet shape entering the squish region after hitting the bowl rim is well predicted both in penetration, width, and equivalence ratio values. In the bowl plane, equivalence ratios penetrate back towards the center of the combustion chamber at $C A=-5.0$, with similar equivalence ratio as the experiment. This highlights correct prediction of the spray injection, impingement and spreading phenomena. The same observations hold also for the highest pressure case, $p_{i n j}=1220 b a r$, as reported in Figure 24. This case shows the best match with the experiments.

A look at the spray cloud structure during the injection is reported in Figure 25: the images highlight formation of a first, initial liquid core early after the 580 start of injection. A few degrees later, at $C A=-20.9 \mathrm{deg}$ aTDC, tiny droplets due to the KH mechanism have already formed across all three jets. Only the highest injection pressure jets however show evidence of RT breakup at the spray tip, where the drop size has been magnified in the picture. RT breakup happens in the $p_{i n j}=500 \mathrm{bar}$ jet one degree later, where the higher pressure jets have already reached the bowl rim and show signs of impingement for some of the RT particles. At $C A=-17.5$ deg aTDC, where the injection event is over for all cases, no signs of coherent liquid structures can be seen, similar to the spray A case, where the liquid length dissipates immediately after the end of 
the injection. A few dribbles remain in the combustion chamber at this point, a kd-tree search structure that reduces the collision calculation time, scaling 

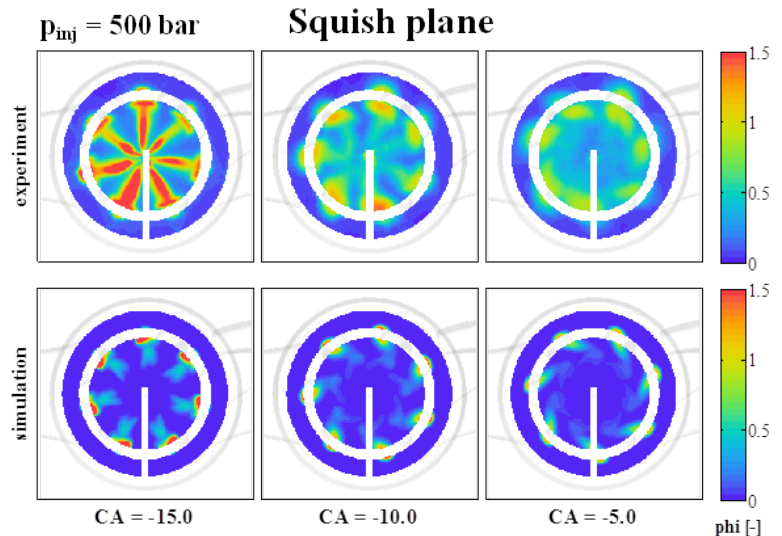

Rim plane
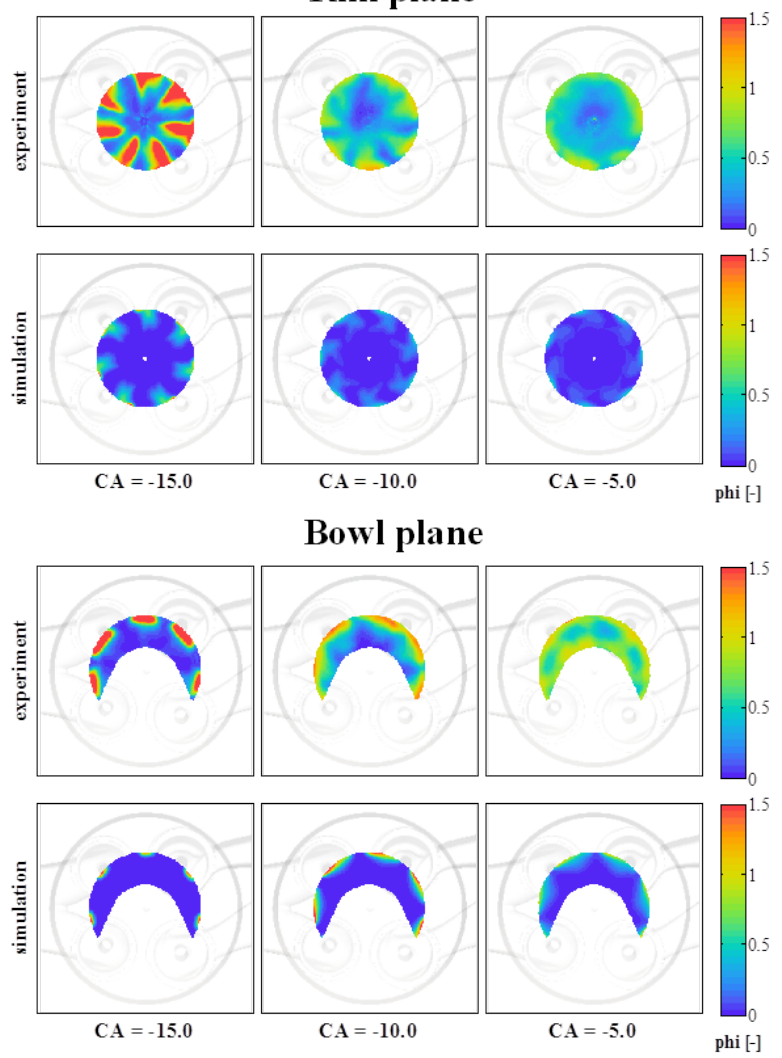

Figure 22: Predicted vs. measured (Sahoo et al. (2011)) in-cylinder equivalence ratio distributions at three planes (squish, rim, bowl) and at three crank angles $(-15,-10,-5$ deg aTDC), for an injection pressure $p_{i n j}=500 \mathrm{bar}$. 

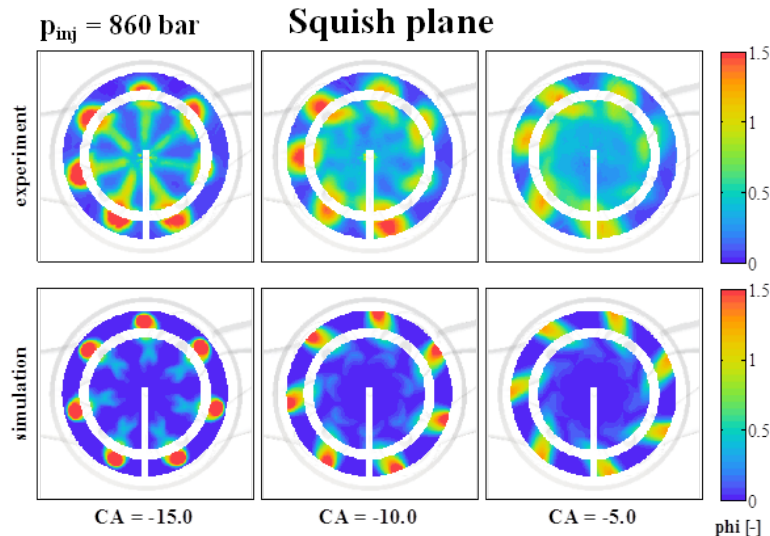

Rim plane
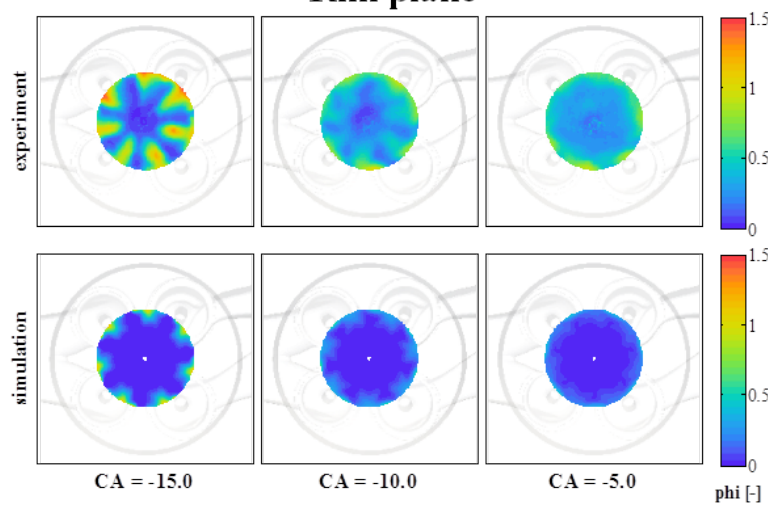

Bowl plane
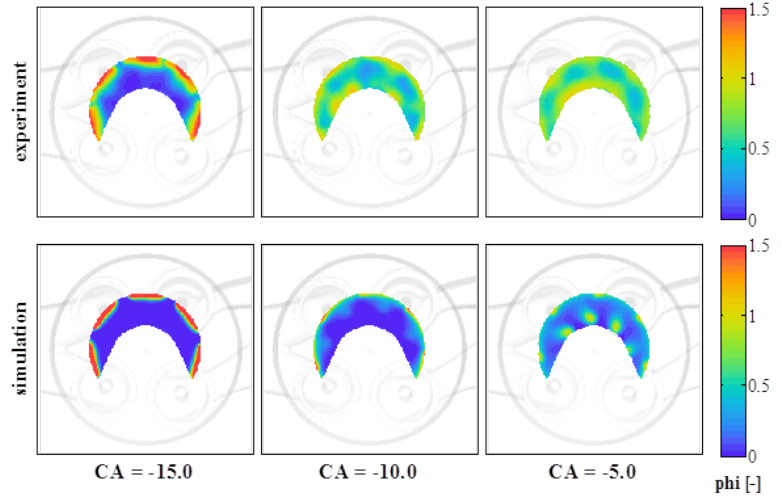

Figure 23: Predicted vs. measured (Sahoo et al. (2011)) in-cylinder equivalence ratio distributions at three planes (squish, rim, bowl) and at three crank angles $(-15,-10,-5$ deg aTDC), for an injection pressure $p_{i n j}=860 \mathrm{bar}$. 

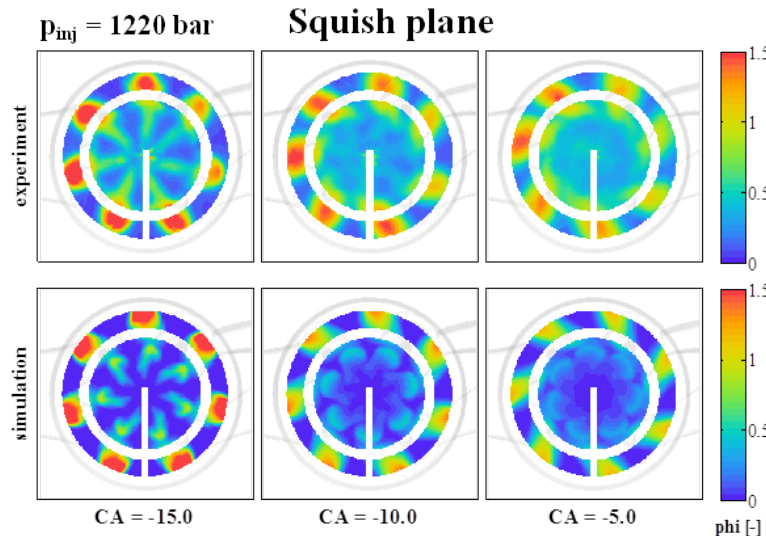

Rim plane
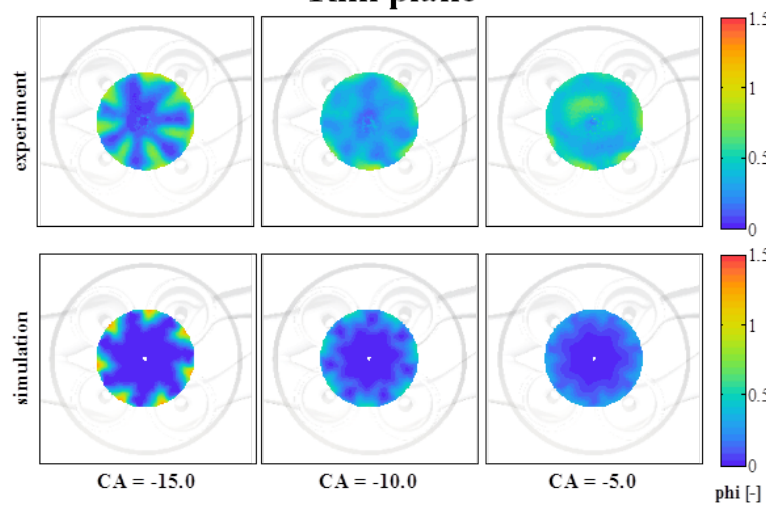

Bowl plane
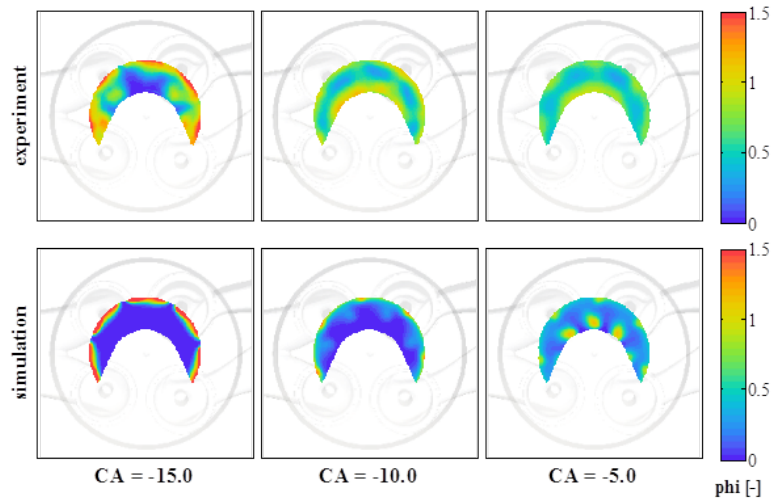

Figure 24: Predicted vs. measured (Sahoo et al. (2011)) in-cylinder equivalence ratio distributions at three planes (squish, rim, bowl) and at three crank angles $(-15,-10,-5$ deg aTDC), for an injection pressure $p_{i n j}=1220 b a r$. 

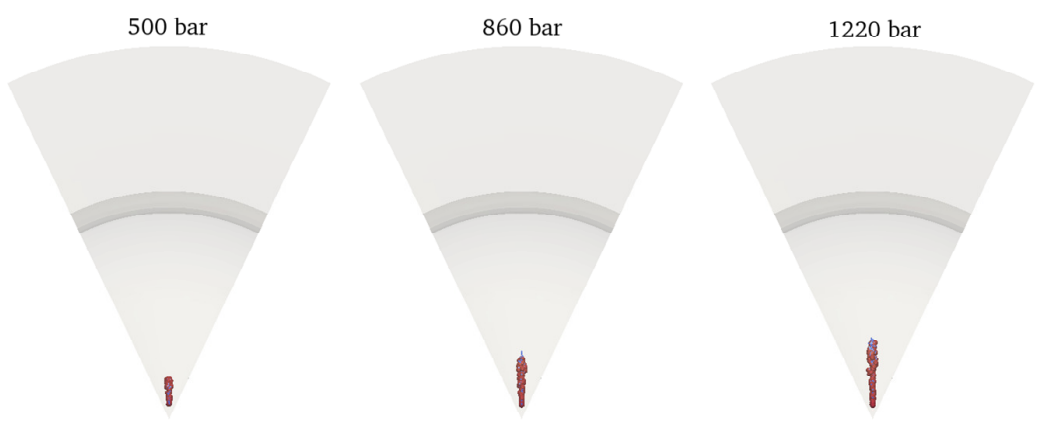

$\mathrm{CA}=-22.5$
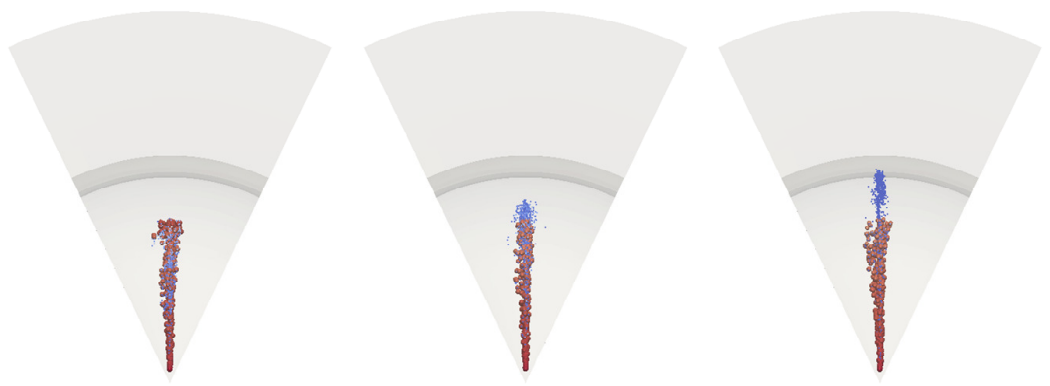

$\mathrm{CA}=-20.9$
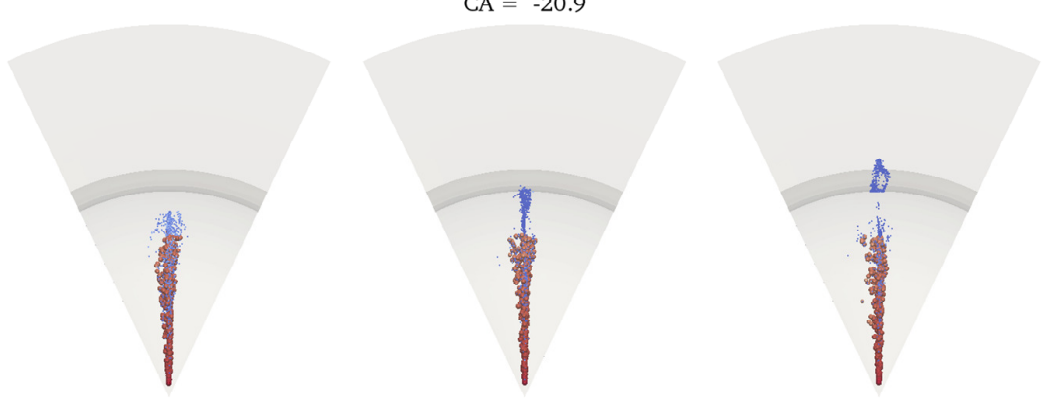

$\mathrm{CA}=-20.0$
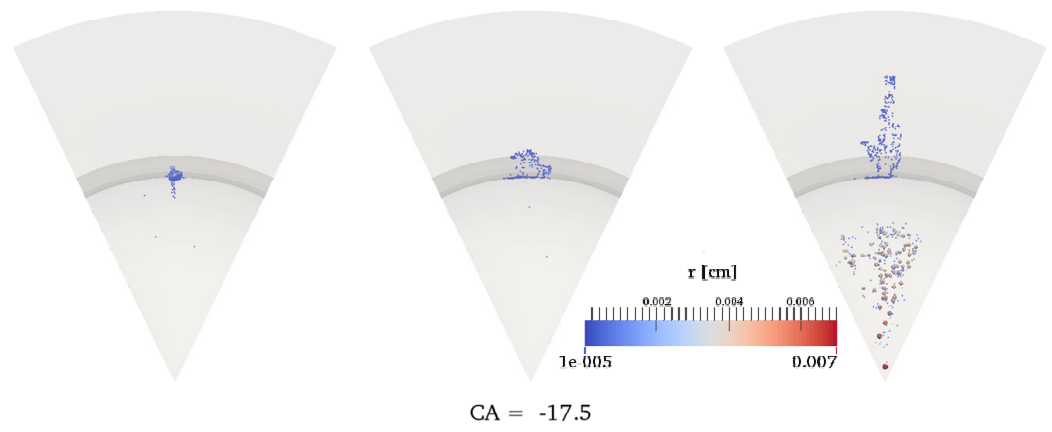

Figure 25: Spray structure comparison for engine simulations with injection pressures (columns) $p_{i n j}=500,860,1220 \mathrm{bar}$, at crank angles (rows) $-22.5,-20,-17.5,-15 \mathrm{deg}$ aTDC. 
linearly with the number of parcels. Finally, an unsteady sub-grid scale flow model for transient spray jets was developed based on the unsteady gas-jet flow model of Abani and Reitz (2007), which exploits a Stokes-Strouhal analogy. The sub-grid scale model was used in the near-nozzle region, instead of the CFDpredicted flow field. This model improves the grid-independency of the spray model avoiding the need to resolve down to nozzle length scales.

Optimal model constants were determined to fit experimental liquid and vapor phase penetration data, as well as gas-phase mixture fraction distributions. Using the same set of model constants, the model was further validated using three mixture preparation experiments carried out on a light-duty optical engine, featuring a single injection pulse at injection pressures of $p_{i n j}=500,860,1220 \mathrm{bar}$. Based on this study, the following conclusions could be drawn:

- the initial spray development transient is directly correlated with global vapor phase penetration indicating the importance of accurate initial transient modeling;

- calibration of the sub-grid scale gas entrainment model parameters suggested a highly responsive Stokes number $S t \approx 0.15$ and a large turbulent entrainment constant $K_{\text {entr }} \approx 0.9$, almost twice the suggested value of Schlichting (2000);

- the effect of the RT breakup model constants was mixed. As Figure 15 shows, the drop sizes after catastrophic breakup are so small that they can be hardly distinguished from the gas-phase in high speed spray experiments;

- parameters in the $\mathrm{KH}$ breakup model converged to the original values suggested by Reitz (1987).

\section{Acknowledgments}

The authors wish to acknowledge support by the Sandia National Laboratories under the U.S. Department of Energy, Office of Vehicle Technologies, 
program managers Leo Breton, Gupreet Singh. Dr. Dipankar Sahoo, Dr. Paul C. Miles and Dr. Stephen Busch are gratefully acknowledged for providing experimental mixture preparation and injection rate data.

\section{References}

Abani, N., Kokjohn, S., Park, S., Bergin, M., Munnannur, A., Ning, W., Sun, Y., Reitz, R.D., 2008a. An Improved Spray Model for Reducing Numerical Parameter Dependencies in Diesel Engine CFD Simulations, in: SAE Technical Paper 2008-01-0970.

Abani, N., Munnannur, A., Reitz, R.D., 2008b. Reduction of numerical parameter dependencies in diesel spray models. Journal of Engineering for Gas Turbines and Power 130, 032809-032809. URL: http://dx.doi.org/10. 1115/1.2830867, doi:10.1115/1.2830867.

Abani, N., Reitz, R.D., 2007. Unsteady turbulent round jets and vortex motion. Physics of Fluids (1994-present) 19,-. URL: http://scitation.aip. org/content/aip/journal/pof2/19/12/10.1063/1.2821910, doi:http:// dx.doi.org/10.1063/1.2821910.

Abraham, J., 1996. Entrapment characteristics of transient gas jets. Numerical Heat Transfer, Part A: Applications 30, 347-364. URL: http://dx. doi.org/10.1080/10407789608913844, doi:10.1080/10407789608913844, arXiv:http://dx.doi.org/10.1080/10407789608913844.

Aggarwal, S., Sirignano, W., 1985. Unsteady spray flame propagation in a closed volume. Combustion and Flame 62,69-84. URL: http://www. sciencedirect.com/science/article/pii/001021808590094X, doi:http: //dx.doi.org/10.1016/0010-2180(85)90094-X.

Amsden, A.A., Orourke, P., Butler, T., 1989. KIVA-2: A computer program for chemically reactive flows with sprays. NASA STI/recon technical report N 89, 27975. 
Beale, J.C., Reitz, R.D., 1999. Modeling spray atomization with the KelvinHelmholtz/Rayleigh-Taylor hybrid model. Atomization and Sprays 9, 623650 .

Bellan, J., 2000. Perspectives on large eddy simulations for sprays: Issues and solutions. Atomization and Sprays 10, 409-425.

Bentley, J.L., 1975. Multidimensional binary search trees used for associative searching. Commun. ACM 18, 509-517. URL: http://doi.acm.org/10. 1145/361002.361007, doi:10.1145/361002.361007.

Bianchi, G.M., Pelloni, P., 1999. Modeling the diesel fuel spray breakup by using a hybrid model, in: SAE Technical Paper 1999-01-0226.

Bremhorst, K., Hollis, P.G., 1990. Velocity field of an axisymmetric pulsed, subsonic air jet. AIAA Journal 28, 2043-2049. URL: http://dx.doi.org/ 10.2514/3.10520, doi:10.2514/3.10520.

Busch, S., 2014. Personal communication.

Butler, T., Cloutman, L., Dukowicz, J., Ramshaw, J., 1979. CONCHAS: An arbitrary Lagrangian-Eulerian computer code for multicomponent chemically reactive fluid flow at all speeds. Technical Report. Los Alamos National Laboratorym LA-8129-MS.

Collins, A., 2014. Cannonball aerodynamic drag. URL: http://arc.id.au/ CannonballDrag.html.

Crowe, C., Schwarzkopf, J., Sommerfeld, M., Tsuji, Y., 1997. Multiphase Flows with Droplets and Particles. Taylor \& Francis.

Cunningham, E., 1910. On the velocity of steady fall of spherical particles through field medium. Proceedings of the Royal Society of London A 83, 357-365. URL: http://www.jstor.org/stable/92978, doi:doi:10.1098/ rspa.1910.0024. 
Deb, K., Pratap, A., Agarwal, S., Meyarivan, T., 2002. A fast and elitist multiobjective genetic algorithm: Nsga-ii. Evolutionary Computation, IEEE Transactions on 6, 182-197. doi:10.1109/4235.996017.

Domingo, P., Vervisch, L., Rveillon, J., 2005. \{DNS $\}$ analysis of partially premixed combustion in spray and gaseous turbulent flamebases stabilized in hot air. Combustion and Flame 140, 172 - 195. URL: http://www.sciencedirect.com/science/article/pii/ S0010218004002342, doi:http://dx.doi.org/10.1016/j.combustflame. 2004.11 .006 .

Dukowicz, J.K., 1980. A particle-fluid numerical model for liquid sprays. Journal of Computational Physics 35, 229 - 253. URL: http://www. sciencedirect.com/science/article/pii/002199918090087X, doi:http: //dx.doi.org/10.1016/0021-9991(80)90087-X.

Estrade, J.P., Carentz, H., Lavergne, G., Biscos, Y., 1999. Experimental investigation of dynamic binary collision of ethanol droplets a model for droplet coalescence and bouncing. International Journal of Heat and Fluid Flow 20, 486 - 491. URL: http://www.sciencedirect. com/science/article/pii/S0142727X99000363, doi:http://dx.doi.org/ 10.1016/S0142-727X (99) 00036-3.

Gorokhovski, M., Herrmann, M., 2008a. Modeling primary atomization. Annual Review of Fluid Mechanics 40, 343366.

URL: http://dx.doi.org/10.1146/annurev.fluid.40. 111406.102200, doi:10.1146/annurev.fluid.40.111406.102200, arXiv:http://dx.doi.org/10.1146/annurev.fluid.40.111406.102200.

Gorokhovski, M., Herrmann, M., 2008b. Modeling primary atomization. Annual Review of Fluid Mechanics 40, 343725 366. URL: http://dx.doi.org/10.1146/annurev.fluid.40. 111406.102200, doi:10.1146/annurev.fluid.40.111406.102200, arXiv:http://dx.doi.org/10.1146/annurev.fluid.40.111406.102200. 
Habchi, C., Verhoeven, D., Huu, C., Lambert, L., Vanhemelryck, J., T.Baritaud, 1997. Modeling atomization and break up in high-pressure diesel sprays, in:

Han, Z., Reitz, R., 1995. Turbulence modeling of internal combustion engines using rng k-epsilon models. Combustion Science and Technology 106, 267-295. URL: http://dx.doi.org/ 10.1080/00102209508907782, doi:10.1080/00102209508907782, arXiv:http://dx.doi.org/10.1080/00102209508907782.

Hiroyasu, H., 2000. Spray breakup mechanism from the hole-type nozzle and its applications. Atomization and Sprays 10, 511-527.

Huh, K.Y., Lee, E., Koo, J., 1998. Diesel spray atomization model considering nozzle exit turbulence conditions. Atomization and Sprays 8, 453-469.

Islam, S.M.N., Tucker, H.J., 1980. Flow in the initial region of axisymmetric turbulent jets. Journal of Fluids Engineering 102, 85-91. URL: http://dx . doi.org/10.1115/1.3240631, doi:10.1115/1.3240631.

Iyer, V., Abraham, J., 1997. Penetration and dispersion of transient gas jets and sprays. Combustion Science and Technology 130, 315-334. URL: http://dx . doi.org/10.1080/00102209708935747, doi:10.1080/00102209708935747, arXiv:http://dx.doi.org/10.1080/00102209708935747.

Launder, B., Spalding, D., 1972. Mathematical Models of Turbulence. Academic Press.

Leboissetier, A., Okongo, N., Bellan, J., 2005. Consistent large-eddy 750 simulation of a temporal mixing layer laden with evaporating drops. part 2. a posteriori modelling. Journal of Fluid Mechanics 523, 3778. URL: http://journals.cambridge.org/article_S0022112004002101, doi:10.1017/S0022112004002101. 
Levich, V., 1962. Physicochemical hydrodynamics: (by) veniamin G. Levich. Transl. by scripta technica, inc. Prentice-Hall international series in the physical and chemical engineering sciences, Prentice-Hall.

Liepmann, D., Gharib, M., 1992. The role of streamwise vorticity in the near-field entrainment of round jets. Journal of Fluid Mechanics 245, 643-668. URL: http://journals.cambridge.org/article_ S0022112092000612, doi:10.1017/S0022112092000612.

Liu, A., Mather, D., Reitz, R., 1993. Modeling the effects of drop drag and breakup on fuel sprays, in: SAE technical paper 930072.

Luo, K., Pitsch, H., Pai, M., Desjardins, O., 2011. Direct numerical simulations and analysis of three-dimensional n-heptane spray flames in a model swirl combustor. Proceedings of the Combustion Institute 33, 2143

- 2152. URL: http://www.sciencedirect.com/science/article/pii/ S1540748910001379, doi:http://dx.doi.org/10.1016/j.proci.2010.06. 077.

Manin, J., Pickett, L., Bardi, M., 2012. Evaluation of the liquid length via diffused back-illumination imaging in vaporizing diesel sprays, in: 8th International Conference on Modeling and Diagnostics for Advanced Engine Systems (COMODIA). URL: http://www.sandia.gov/ecn/cvdata/assets/ movies/bkldaAL1movie.php.

Meijer, M., Somers, B., Johnson, J., Naber, J., Lee, S.Y., Malbec, L.M., Bruneaux, G., Pickett, L.M., Bardi, M., Payri, R., Bazyn, T., 2012. Engine CombustionNetwork (ECN): Characterization and Comparison of Boundary Conditions for Different Combustion Vessels. Atomization and Sprays 22, $777-806$.

Miles, P.C., Sahoo, D., Busch, S., Trost, J., Leipertz, A., 2013. 780 Pilot injection ignition properties under low-temperature, dilute in-cylinder conditions. SAE International Journal of Engines 6, 1888-1907. URL: http://saeeng.saejournals. 
org/content/6/4/1888.abstract,

doi:10.4271/2013-01-2531, arXiv:http://saeeng. saejournals.org/content/6/4/1888.full.pdf+html.

Miller, D.G., Bailey, A.B., 1979. Sphere drag at mach numbers from 03 to 20 at reynolds numbers approaching 107. Journal of Fluid Mechanics 93, 449-464. URL: http://journals.cambridge.org/article_ S0022112079002597, doi:10.1017/S0022112079002597.

Miller, R.S., Bellan, J., 2000. Direct numerical simulation and subgrid analysis of a transitional droplet laden mixing layer. Physics of Fluids (1994-present) 12, 650-671. URL: http://scitation.aip.org/content/ aip/journal/pof2/12/3/10.1063/1.870271, doi:http://dx.doi.org/10. 1063/1.870271.

Morrison, F.A., 2013. Data correlation for drag coefficient for sphere. URL: www . chem.mtu . edu/ fmorriso/DataCorrelationForSphereDrag2013.pdf.

Munnannur, A., Reitz, R.D., 2007. A new predictive model for fragmenting and non-fragmenting binary droplet collisions. International Journal of Multiphase Flow 33, 873 - 896. URL: http://www.sciencedirect. com/science/article/pii/S0301932207000419, doi:http://dx.doi.org/ 10.1016/j.ijmultiphaseflow.2007.03.003.

Munnannur, A., Reitz, R.D., 2009. A comprehensive collision model for multidimensional engine spray computations. Atomization and Sprays 19, $597-$ 619. doi:http://dx.doi.org/10.1615/AtomizSpr.v19.i7.10.

Musculus, M.P., 2009. Entrainment waves in decelerating transient turbulent jets. Journal of Fluid Mechanics 638, 117-140. URL: http: //journals.cambridge.org/article_S0022112009990826, doi:10.1017/ S0022112009990826.

Musculus, M.P., Miles, P.C., Pickett, L.M., 2013. Conceptual models for partially premixed low-temperature diesel combustion . Progress in Energy and Combustion Science 39, 246 - 283. URL: http://www. sciencedirect. 
com/science/article/pii/S0360128512000548, doi:http://dx.doi.org/ $10.1016 / j \cdot \operatorname{pecs} .2012 .09 .001$.

Okongo, N., Bellan, J., 2000. A priori subgrid analysis of temporal mixing layers with evaporating droplets. Physics of Fluids (1994-present) 12, 15731591. URL: http://scitation.aip.org/content/aip/journal/pof2/12/ 6/10.1063/1.870405, doi:http://dx.doi.org/10.1063/1.870405.

Okongo, N., Bellan, J., 2004. Consistent large-eddy simulation of a temporal mixing layer laden with evaporating drops. part 1. direct numerical simulation, formulation and a priori analysis. Journal of Fluid Mechanics 499, 147. URL: http://journals.cambridge.org/article_S0022112003007018, doi:10.1017/S0022112003007018.

O'Rourke, P.J., 1981. Collective drop effects on vaporizing liquid sprays. Ph. D. Dissertation, Dept. Mech. Aerospace Engg., Princeton University.

Pera, C., Rveillon, J., Vervisch, L., Domingo, P., 2006. Modeling subgrid scale mixture fraction variance in $\{\mathrm{LES}\}$ of evaporating spray. Combustion and Flame 146, 635 - 648. URL: http://www.sciencedirect. com/science/article/pii/S0010218006001623, doi:http://dx.doi.org/ $10.1016 / \mathrm{j}$. combustflame.2006.07.003.

Perini, F., Dempsey, A., Reitz, R., Sahoo, D., Petersen, B., Miles, P., 2013. A Computational Investigation of the Effects of Swirl Ratio and Injection Pressure on Mixture Preparation and Wall Heat Transfer in a Light-Duty Diesel Engine. SAE technical paper 2013-01-1105 URL: http://dx.doi. org/10.4271/2013-01-1105, doi:10.4271/2013-01-1105.

Perini, F., Miles, P.C., Reitz, R.D., 2014. A comprehensive modeling study 835 of in-cylinder fluid flows in a high-swirl, light-duty optical diesel engine . Computers \& Fluids 105, 113 - 124. URL: http://www.sciencedirect. com/science/article/pii/S004579301400348X, doi:http://dx.doi.org/ 10.1016/j.compfluid.2014.09.011. 
Pickett, L.M., Manin, J., Genzale, C.L., Siebers, D.L., Musculus, M.P.B.,

Idicheria, C.A., 2011. Relationship between diesel fuel spray vapor penetration/dispersion and local fuel mixture fraction. SAE Int. J. Engines 4, 764-799. URL: http://dx.doi.org/10.4271/2011-01-0686, doi:10.4271/ 2011-01-0686.

Pope, S.B., 1978. An explanation of the turbulent round-jet/plane-jet anomaly. AIAA Journal 16, 279-281. URL: http://dx.doi.org/10.2514/3.7521, doi:10.2514/3.7521.

Ra, Y., Kong, S.C., Reitz, R.D., Rutland, C.J., Han, Z., 2005. Multidimensional modeling of transient gas jet injection using coarse computational grids, in: SAE Technical Paper 2005-01-0208. sprays. Atomisation Spray Technology 3, 309 - 337.

Reitz, R.D., Bracco, F., 1979. On the dependence of spray angle and other spray pparameter on nozzle design and operating conditions, in: SAE Technical Paper 790494. doi:doi : 10.4271/790494.

Reitz, R.D., Bracco, F.V., 1982. Mechanism of atomization of a liquid jet. Physics of Fluids (1958-1988) 25, 1730-1742. URL: http://scitation.aip. org/content/aip/journal/pof1/25/10/10.1063/1.863650, doi:http:// dx.doi.org/10.1063/1.863650.

Reitz, R.D., Diwakar, R., 1987. Structure of high-pressure fuel sprays, in: SAE Technical Paper 870598. doi:doi:10.4271/870598.

Reitz, R.D., Duraisamy, G., 2015. Review of high efficiency and clean reactivity controlled compression ignition (rcci) combustion in internal combustion engines. Progress in Energy and Combustion Science 46, 12 - 71. URL: http://www.sciencedirect.com/science/article/ 865 pii/S0360128514000288, doi:http://dx.doi.org/10.1016/j.pecs. 2014. 05.003 . 
Reveillon, J., Bray, K., Vervisch, L., 1998. Dns study of spray vaporization and turbulent micro-mixing, in: Aerospace Sciences Meetings. American Institute of Aeronautics and Astronautics, pp. -. URL: http://dx.doi.org/10.2514/ 6.1998-1028, doi:10.2514/6.1998-1028.

Reveillon, J., Vervisch, L., 2005. Analysis of weakly turbulent dilutespray flames and spray combustion regimes. Journal of Fluid Mechanics 537, 317-347. URL: http://journals.cambridge.org/article_ S0022112005005227, doi:10.1017/S0022112005005227.

Sahoo, D., Miles, P.C., Trost, J., Leipertz, A., 2013. The impact of fuel mass, injection pressure, ambient temperature, and swirl ratio on the mixture preparation of a pilot injection. SAE International Journal of Engines 6, 1716-1730. URL: http://saeeng.saejournals. org/content/6/3/1716. abstract, doi:10.4271/2013-24-0061, arXiv:http://saeeng. saejournals.org/content/6/3/1716.full.pdf+html.

Sahoo, D., Petersen, B., Miles, P., 2011. Measurement of equivalence ratio in a light-duty low temperature combustion diesel engine by planar laser induced fluorescence of a fuel tracer. SAE International Journal of Engines 4, 2312-2325. URL: http://saeeng.saejournals. org/content/4/2/2312. abstract, doi:10.4271/2011-24-0064, arXiv:http://saeeng. saejournals.org/content/4/2/2312.full.pdf+html.

Sahoo, D., Petersen, B., Miles, P., 2012. The impact of swirl ratio and injection pressure on fuel-air mixing in a light-duty diesel engine, in: ASME 2012 Internal Combustion Engine Division Spring Technical Conference ICES2012.

Sandia, 2014. Sandia engine combustion network. URL: http://www. sandia. gov/ecn.

Sankaran, V., Menon, S., 2002. Les of spray combustion in swirling flows. Journal of Turbulence 3, N11. URL: http://dx.doi. org/10.1088/1468-5248/3/1/011, doi:10.1088/1468-5248/3/1/011, arXiv:http://dx.doi.org/10.1088/1468-5248/3/1/011. 
Schlichting, H., 2000. Boundary-Layer Theory. Physics and astronomy, Springer.

URL: http://books . google.it/books?id=8YugVtom1y4C.

Schmidt, D.P., Rutland, C.J., 2004. Reducing grid dependency in droplet collision modeling. Journal of Engineering for Gas Turbines and Power 126, 227-233. URL: http://dx.doi.org/10.1115/1.1564066, doi:10.1115/1. 1564066 .

Singh, S., Musculus, M.P.B., 2010. Numerical modeling and analysis of entrainment in turbulent jets after the end of injection. Journal of Fluids Engineering 132, 081203-081203. URL: http://dx.doi.org/10.1115/1.4002184, doi:10.1115/1.4002184.

Song, L., Abraham, J., 2003. Entrainment characteristics of transient turbulent round, radial and wall-impinging jets: Theoretical deductions. Journal of Fluids Engineering 125, 605-612. URL: http://dx.doi.org/10.1115/1. 1593707, doi:10.1115/1.1593707.

Tanner, F., 1997. Liquid jet atomization and droplet breakup modeling of nonevaporating diesel fuel sprays, in: SAE Technical Paper 970050. doi:doi: $10.4271 / 970050$.

Torres, D.J., O'rourke, P.J., Amsden, A.A., 2003. Efficient multicomponent fuel algorithm. Combustion Theory and Modelling 7, 66-86. URL: http://dx. doi.org/10.1088/1364-7830/7/1/304, doi:10.1088/1364-7830/7/1/304, arXiv:http://dx.doi.org/10.1088/1364-7830/7/1/304.

Torres, D.J., Trujillo, M.F., 2006. KIVA-4: An unstructured ALE code for compressible gas flow with sprays. Journal of Computational Physics 219, 943 - 975. URL: http://www.sciencedirect. com/science/article/pii/S002199910600338X, doi:http://dx.doi.org/ $10.1016 / j \cdot j c p .2006 .07 .006$.

Tsang, C.W., Trujillo, M.F., Rutland, C.J., 2014. Large-eddy simulation of shear flows and high-speed vaporizing liquid fuel sprays. Computers \& Fluids 
105, 262 - 279. URL: http://www.sciencedirect.com/science/article/ pii/S004579301400351X, doi:http://dx.doi.org/10.1016/j.compfluid. 2014.09 .014$.

Wang, Y., Ge, H.W., Reitz, R.D., 2010. Validation of Mesh- and Timestep- Independent Spray Models for Multi-Dimensional Engine CFD Simulation. SAE International Journal of Fuels and Lubricants 3, 277-302. URL: http://saefuel.saejournals. org/content/3/1/277. abstract, doi:10.4271/2010-01-0626, arXiv:http: //saefuel. saejournals.org/content/3/1/277.full.pdf+html. 